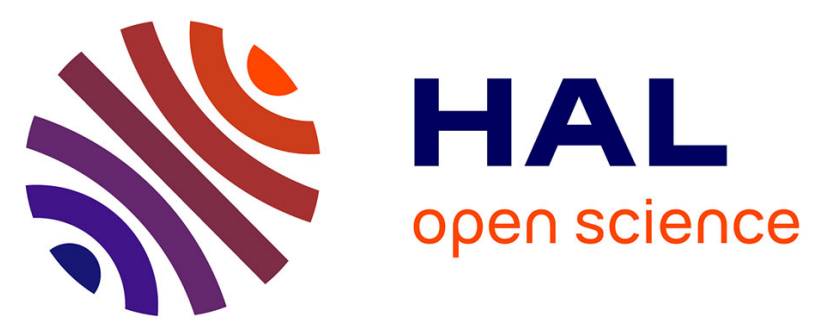

\title{
Free-Form Deformation Digital Image Correlation (FFD-DIC): a non-invasive spline regularization for arbitrary finite element measurements
}

Morgane Chapelier, Robin Bouclier, Jean-Charles Passieux

\section{- To cite this version:}

Morgane Chapelier, Robin Bouclier, Jean-Charles Passieux. Free-Form Deformation Digital Image Correlation (FFD-DIC): a non-invasive spline regularization for arbitrary finite element measurements. Computer Methods in Applied Mechanics and Engineering, 2021, 384, pp.113992. 10.1016/j.cma.2021.113992 . hal-03273312

\section{HAL Id: hal-03273312 \\ https://hal.science/hal-03273312}

Submitted on 29 Jun 2021

HAL is a multi-disciplinary open access archive for the deposit and dissemination of scientific research documents, whether they are published or not. The documents may come from teaching and research institutions in France or abroad, or from public or private research centers.
L'archive ouverte pluridisciplinaire HAL, est destinée au dépôt et à la diffusion de documents scientifiques de niveau recherche, publiés ou non, émanant des établissements d'enseignement et de recherche français ou étrangers, des laboratoires publics ou privés. 


\section{Highlights}

Free-Form Deformation Digital Image Correlation (FFD-DIC): a non-invasive spline regularization for arbitrary finite element measurements.

M. Chapelier, R. Bouclier, J.-C. Passieux

- A free-form-deformation-based regularization is proposed for general FE measurement in DIC.

- An arbitrary FE mesh is embedded into a B-spline box and the corresponding FE dof are related to the control variables attached to the box.

- The method can be interpreted as a projection onto a reduced, more regular, basis and is non-invasive w.r.t. FEM.

- A specific care is taken to handle conditioning problems and a careful additional projection onto local directions is performed for robustness in the full 3D case.

- The performance of the method is demonstrated over real images for FE displacement and shape measurements in DIC and stereo-DIC. 


\title{
Free-Form Deformation Digital Image Correlation (FFD-DIC): a non-invasive spline regularization for arbitrary finite element measurements.
}

\author{
M. Chapelier ${ }^{\mathrm{a}, \mathrm{b}, *}$, R. Bouclier ${ }^{\mathrm{a}, \mathrm{b}}$, J.-C. Passieux ${ }^{\mathrm{a}}$ \\ ${ }^{a}$ Institut Clément Ader (ICA), Université de Toulouse, CNRS-INSA-UPS-ISAE-Mines Albi, Toulouse, \\ France \\ ${ }^{b}$ Institut de Mathématiques de Toulouse (IMT), Université de Toulouse, CNRS-INSA-UT1-UT2-UPS, \\ Toulouse, France
}

\begin{abstract}
Regularizing Finite-Element Digital Image Correlation (FE-DIC) problems is essential when using fine meshes, for instance to get a direct bridge with simulation tools or in view of performing data assimilation. Shape optimization brings comparable concerns so it is proposed to draw inspiration from this field of research. In this respect, a new general spline-based non-invasive regularization scheme is proposed, based on the Free-Form Deformation (FFD) concept that offers the opportunity to decouple the design space from that of the actual geometry to be updated. The main idea is to embed the input FE mesh, with any element type or size, into a simple cuboid B-spline box and to relate the nodal FE dof of the measured field to another more regular field discretized by the box. The regularization length is thus directly driven by the box refinement. A specific care is taken to handle possible conditioning problems caused by non-influential control points. From a practical point of view, the method can be interpreted as a projection on a reduced basis; therefore, it results in a regularized but conventional FE measured field which can be simply computed from a standard FE-DIC code. The developed scheme is applied for FE displacement measurement using DIC and for mesh-based measurement in stereo-DIC. For the latter, a novel procedure based on additional projections over local directions complements the FFD method for full efficiency. The performance of the strategy is assessed on real images coming from various experimental tests and comparisons are made with other published techniques to prove its
\end{abstract}


effectiveness.

Keywords: optimization, image registration, mesh-based shape measurement, stereo-correlation, multilevel B-splines, reduced-order modeling.

\section{Introduction}

Digital Image Correlation (DIC) denotes in the field of experimental solid mechanics what is also referred to as image registration in the computer vision and applied mathematics communities. DIC has become one of the most commonly used techniques to obtain fullfield measurements on structures, because of its simplicity (it is non-contact and makes use of multipurpose reusable hardware) and its modularity (no intrinsic physical scale). Depending on the imaging devices, it is possible to derive 2D surface, 3D surface, or even 3D volume displacement fields by taking pictures of a structure at different loading states and solving an inverse problem. The associated method can be classified as 2D-DIC (or simply DIC) $[44,70,69,26,20,77]$, stereo-DIC [71, 23, 15, 56, 2] and Digital Volume Correlation (DVC) $[4,41,24]$, respectively. In the case of stereo-DIC, a similar set up allows to measure the shape of a 3D mechanical surface $[5,16,56,13]$, which is actually a fundamental step prior to any experiment for 3D surface displacement field measurements. The general DIC approach finds numerous applications in engineering: material characterization, geometry control, defect identification, model validation, etc.

Among multiple variants of the method, Finite-Element (FE) mesh-based techniques (also named global- or FE-DIC) $[69,6,26,20,77,56,52]$ have widely spread because of their ability to be directly coupled with simulation software in view of performing data assimilation [54, 59, 47]. The measured field is indeed sought in the space generated by the FE functions associated to the FE mesh that is used for computational mechanics. Consequently, FE meshes are generally quite fine to accurately capture the solution over structures of potentially complex geometries. In this paper, such meshes that are designed

\footnotetext{
*Institut Clement Ader (ICA), Université de Toulouse, CNRS-INSA-UPS-ISAE-Mines Albi, 135 avenue de Rangueil, 31077 Toulouse, France. E-mail: chapelie@insa-toulouse.fr
} 
for accurate simulation results will be referred to as analysis-suitable meshes. When directly used to describe the kinematic measured field in DIC, they usually lead to ill-posed problems since the meshes contain too many degrees of freedom (dof) to optimize compared to the data provided by the experimental instrumentation (camera resolution, speckle pattern resolution, etc). Such a problematic is even more exacerbated in case of mesh-based shape measurement since the shape of the specimen is generally rather smooth and regular, and thus should require less dof than the corresponding simulated displacement field which may comprise important gradients [13]. To tackle this issue in FE-DIC, the common practice consists in resorting to what is referred to as the Tikhonov regularization technique in the field, i.e. to add a specific term to the initial DIC objective function that penalizes the $L_{2}$-norm of the gradient of each component of the measured field $[50,56,16]$. Yet, this treatment may create important artefacts, especially when the kinematic transformation involves global rotations [13].

Shape optimization brings comparable concerns so it is proposed to draw inspiration from this field of research to improve the general area of DIC. Indeed, dealing with a large number of dof causes unrealistic geometries, and therefore the use of FE dof as design variables is usually combined with the use of smoothing filters [40, 21, 7, 29]. Another classic approach, sometimes referred to as CAD-based approach, consists in considering a CAD model, i.e. made of spline functions (typically B-splines or NURBS $[12,55]$ ), to define the design variables $[10,49,75,37,36]$. The spline functions are obviously well suited for shape optimization since they have been built for geometric modeling in CAD and computer graphics; they are of higher regularity and thus imply few dof (mainly associated to the control point positions of the spline entities) to describe a geometry and, more importantly, a geometry update.

Taking the reasoning even further, the spline technology actually provides a natural regularization framework for general optimization problems since it allows to look for the solution in a more regular approximation subspace. In this respect, some work has been performed to regularize DIC problems using B-spline and NURBS functions. First, the ability of B-spline functions to accurately measure displacement fields along with their derivatives 
in 2D-DIC was established $[11,18,38]$. Then, B-spline functions were also used for smooth 3D shape measurements $[5,16]$ before being investigated for real 3D surface displacement measurements [15] in the context of stereo-DIC. From a global point of view, all these methods are similar to FE-DIC, but instead of using FE basis functions, they consider B-spline functions to describe the specimen geometry, the sought measured fields, and hence to compute all the operators needed for the resolution. As a result, besides the effort to implement spline functions in the DIC framework, the user ends up with an experimental displacement field that is not directly comparable with the output provided by most of today's simulation tools, which was the core interest of global approaches in DIC. An attempt to keep fields defined on FE meshes while taking advantage of the increased smoothness of B-Spline and NURBS functions has more recently been performed for shape measurements in [13]. The purpose was to make use of the explicit (Bézier-based) link from Lagrange nodal polynomials to spline functions $[9,66,73]$ to project the initial FE shape measurement problem onto a smoother spline one. This technique is interesting since it allows to measure a 3D spline shape while using a standard FE-DIC software in a non-invasive way; however, it is restricted to input FE meshes of which approximation subspaces include the initial CAD space. In practice, this means that the FE mesh needs to be at least quadratic and even made of elements exhibiting a tensor product structure, which is usually not the case for any arbitrary analysis-suitable mesh. Another issue with all the previously mentioned strategies is the construction of the input proper boundary-fitted spline parametrization of the specimen geometry from CAD data, which is not a trivial task as demonstrated by the recent developments in isogeometric analysis [46, 72]. Here, we place ourselves from the experimental mechanics engineer point of view who needs to perform a relevant field measurement starting from a given FE mesh coming from a simulation software. In other words, the objective is to develop a new non-invasive regularization scheme that can be applied to any FE mesh, regardless of the element size, type or spatial density.

In order to do so, we propose in this paper to make use of the Free-Form Deformation (FFD) concept that was first introduced by Sederberg and Parry [67] in the field of computer graphics and later applied in engineering fields, in particular for the aerodynamic design of 
structures $[1,17,34,39,29,35,45]$ or to more general shape optimization problems [8, 27], to make structures fit given data points in mechanical and biomedical engineering [3, 63, 64], and for non-rigid image registration in medical imaging [62, 78, 76, 68, 33]. The FFD approach consists in embedding an object - respectively, a CAD geometric model in computer graphics, a mechanical structure in shape optimization or in data fitting, or an image in medical imaging - into a morphing box (usually made of splines). Any deformation inside the morphing box is then only described by the deformation of the morphing box itself. In this respect, note that what is classified as the FFD approach in medical imaging is actually very similar to the direct spline DIC method discussed previously [18, 38] in the sense that both techniques seek to find a spline displacement field that matches different images. In contrast, we have to consider in this study, as embedded object, a mechanical structure that is described by its analysis-suitable FE mesh. Thus our situation is closer to what is encountered in FFD shape optimization $[34,45,8]$ in terms of definition of the design space. The FFD concept offers the opportunity to decouple the design space from that of the actual geometry to be updated. As a result, even if the geometry is not regular $\left(e . g ., C^{0}\right.$ across elements in case of a FE mesh), the deformation of the geometry can be considered of increased smoothness so as to regularize the corresponding optimization problem. The key idea here is just to link each FE nodal dof of the field of interest to another, more regular field discretized by the morphing box. As such, the initial structure of the FE mesh is kept during the whole process and we end up with a conventional measured FE field. Furthermore, the method is non-invasive with respect to a standard FE code (classic quadrature rules, element connectivities, etc). The strategy can ultimately be interpreted as a projection on a reduced, smooth basis and actually consists in a generalization of the technique introduced in [13]. Indeed, any FE mesh can be embedded in a morphing box and thus any FE field dof can be controlled by the deformation of the morphing box.

The presented work is organized as follows: after this introduction, section 2 dwells on theoretical and numerical aspects of the proposed FFD scheme. The adopted choices and innovative treatments with respect to the general field of FFD to build the morphing box and to relate the FE field of interest to the deformation of the box are clearly accounted for and 
highlighted. In particular, we consider a simple cuboid spline box and thus need to develop a specific strategy to handle conditioning problems caused by non-influential control points. In addition, we provide an original point of view on the developed FFD method and discuss its connection with other existing techniques, namely projection methods $[58,73,13,19]$ and fictitious domain approaches [57, 43, 65, 25]. Then, our FFD method is applied in section 3 to regularize 2D-DIC for FE displacement field measurements. Note as of now that the extension to DVC could be straightforwardly performed. The minimization problem and the associated resolution are detailed before the performances of the method are demonstrated on real images taken to observe the bending of a $2 \mathrm{D}$ beam. Then, section 4 is devoted to the delicate problem of FE mesh shape measurement. Innovative developments based on additional projections over local directions are performed to adapt to complex geometries. The results coming from real images of a twisted plate and of a spherical cap are given to assess the methodology. Finally, concluding remarks are drawn in section 5 .

\section{Free-Form Deformation projection}

As most of the works dealing with FFD, we use B-spline functions to create the morphing box. NURBS functions could also be considered [76]. Yet the B-spline technology is the easiest and most encountered one and appears sufficient for the applications carried out in this work. The analysis-suitable FE mesh will thus be controlled by a simple embedding B-spline box deformation. One can already see that this method is of great interest when the structure geometry is complex, described by a fine and possibly unstructured FE mesh, since the box geometry can be chosen very simply and with a number of dof adapted to the deformation to describe. First, some general and useful properties of B-spline functions are given in this section. Then, the creation of the morphing box is outlined, before the link between the deformation of the FE mesh and the one of the morphing box is properly derived. Finally, some insights regarding the implementation are given along with a discussion on good practices to avoid bad conditioning. 


\subsection{B-splines: basics}

B-spline functions are commonly used in CAD. These piecewise polynomial functions are smooth and thus few dof are needed to generate a representative geometry thanks to these functions. The B-spline technology is now relatively well-known in the computational mechanics community, in particular with the advent of isogeometric analysis [31, 14]. Only the fundamentals are given here. For further details, the interested reader is referred to the works cited hereafter.

Univariate B-spline functions are defined in a 1D parametric domain, which is divided into elements (or knot spans) thanks to knots. Some knots can be repeated in order to lower the regularity of the functions at these knots. In this article, for the sake of simplicity, the parametric domain will be an interval $I=[0, L]$, where $L$ is directly chosen as characteristic length in the physical space, and the knot-vector will be open (first and last knot of the interval are repeated $p+1$ times, $p$ being the functions degree) and uniform (the intervals delimited by two successive knots are of same length and no other knots than those on the edges are repeated). The domain delimitated by the first and the last knot is called a patch. Simple geometries can be modeled with a single patch, which will be our case in this work where the generation of a single box will be sufficient. From the knots, univariate B-spline functions are defined recursively depending on their degree, and efficiently computed thanks to the Cox-de Boor algorithm $[12,55]$. Multivariate B-splines, dimension of which is greater than 1, are simply constructed with a tensor product of univariate B-splines.

A $\delta$-dimensional structure $\underline{\mathcal{S}}$ living in a $D$-dimensional space is then defined as:

$$
\begin{aligned}
\underline{\mathcal{S}}: I \subset \mathbb{R}^{\delta} & \longrightarrow \mathbb{R}^{D} \\
\underline{\xi} & \longmapsto \underline{\mathcal{S}}(\underline{\xi})=\underline{X}=\sum_{i=1}^{n_{I G}} N_{i}(\underline{\xi}) \underline{P}_{i},
\end{aligned}
$$

where $\xi$ is a parameter living in $I$ the parametric domain, and $\left(N_{i}\right)_{i=1 . . n_{I G}}$ are the $p^{\text {th }}$ degree $\delta$-variate spline functions associated to the $n_{I G}$ control points $\left(\underline{P}_{i}\right)_{i=1 . . n_{I G}}$ that have $D$ coordinates in the physical space. $\underline{X}$ is the image of $\underline{\xi}$ in the physical domain. The structure $\underline{\mathcal{S}}$ thus created is composed of a one-patch spline geometry. Note that $D \geqslant \delta$. A matrix form is straightforward for each dimension $k$ of the physical domain: 


$$
\mathcal{S}_{k}(\underline{\xi})=X_{k}=\underline{N(\underline{\xi})^{T}} \underline{P_{k}}, \quad \forall k \in 1 . . D
$$

where the $i^{t h}$ component of vector $N(\underline{\xi})$, i.e. $\{N(\underline{\xi})\}_{i}$, is equal to $N_{i}(\underline{\xi}), X_{k}$ is the $k^{t h}$ coordinate of $\underline{X}$ and $\underline{P_{k}}$ is a vector such that $\left\{\underline{P_{k}}\right\}_{i}=\underline{P}_{i_{k}}$.

B-spline objects are very smooth. Indeed, they are $(p-m)$-continuous at knots of multiplicity $m$. To keep a reasonable support size for the functions and thus allow to describe local variations of the field of interest, we choose a low degree ( $p=2$ in this article). Nevertheless, in order to achieve maximum regularization, we take interior knots of multiplicity $m=1$. Therefore, B-splines quantities are $C^{1}$-continuous in this paper.

Another major advantage of B-spline functions is their refinement procedures. In particular, it is possible to add any number of knots with a simple algorithm and make this refinement keep the geometry unchanged. This refinement procedure, called knot insertion, can be explicitly written as a matrix link $\underline{\underline{C}}_{r e f}$ between the B-spline functions of the coarse geometry $\underline{\mathcal{S}}_{\text {coarse }}$ and the B-spline functions of the fine geometry $\underline{\mathcal{S}}_{\text {fine }}$. Prescribing the nonmodification of the geometry, the same matrix also allows to link the position of the control points of the coarse geometry with those of the fine geometry. More precisely, the relations read:

$$
\begin{aligned}
\underline{N(\underline{\xi})}_{\text {coarse }} & =\underline{\underline{C}}_{\text {ref }} \underline{N(\underline{\xi})}_{\text {fine }}, \\
\underline{P}_{\text {fine }} & =\underline{\underline{C}}_{\text {ref }}^{T} \underline{P}_{\text {coarse }} .
\end{aligned}
$$

This advantage allows using a simple and flexible multilevel approach, which has been initially developed for shape optimization (see, e.g., $[48,37,36,28]$ ) and then adapted to multiscale DIC initialization procedures in [13]. Such a multilevel approach will be used in this article that also undertakes to solve some sort of optimization problems (see sections 3 and 4). It consists in first creating a coarse mesh to get the principal solution field features at the first iterations of the optimization process. It is then refined to capture the higher frequency part of the solution field. B-spline functions can also be order-elevated while still keeping the same geometry (degree-elevation process). This process will not be used in the 
multilevel approach here since we only use quadratic B-splines. More information about knot insertion and degree elevation, as well as further details about spline functions can be found, for instance, in $[55,42,14]$.

\subsection{Creating the morphing box}

In the rest of the article, the morphing box geometry was chosen to be a one-patch Bspline $\delta$-orthotope entity, i.e. a rectangle if $\delta=2$ or a rectangular parallelepiped if $\delta=3$. The choice of $\delta$ will depend on the structure geometry, and we have $\delta \leqslant D$ (see Fig. 1 that will be further explained in the following). These geometries are particularly easy to create because the parametric domain $I$ can be chosen so that the mapping from $I$ to the physical domain is the identity function [64]. This is what is performed in this work, and one of the reasons that led us to use B-splines rather than NURBS. However, the defect with such a simple geometric modeling is that some morphing box control points may not have an influence on the embedded FE mesh because they are too far from the latter, in other words, because the support of the corresponding basis function does not, or only slightly, intersect the FE mesh. This issue, which is expected to lead to ill-conditioning, will be addressed in section 2.5. To circumvent the problematic, most of other works preferred morphing boxes that are closer to the structure, but therefore have a mapping that is not the identity function $[1,17,34,45]$. In this case, a step is needed to determine the position of the embedded geometry in the parametric domain of the box (inversion of a possibly non-linear mapping). The dimensions of the morphing box are defined by the embedded structure geometry so that it is tangent to some parts of the structure (see Fig. 1 (left) for illustration). To construct the morphing box, a simple one-element B-spline box is first created and then, automatic refinement procedures are used to get to the desired degree and number of elements in each dimension. The morphing box is finally deformed by modifying the control points locations $\left(\underline{P}_{i}\right)_{i}$ in the physical domain of dimension $D$ (see Fig. 1 (right)). In the following, the link between the deformation of the morphing box and that of the embedded FE mesh will be explained. 


\subsection{Linking the deformation of the FE mesh to that of the morphing box}

The FE mesh deformation is completely driven by the morphing box deformation. As stated previously, the morphing box is defined with B-splines and thus verifies Eq. (1). Let us denote by $n_{F F D}$ the number of control points of the morphing box. In this article, we recall that the embedded structure is described by a FE mesh:

$$
\underline{\mathcal{M}}(\underline{x})=\sum_{j=1}^{n_{F E}} L_{j}(\underline{x}) \underline{X}_{j}^{F E},
$$

where $n_{F E}$ is the number of $\mathrm{FE}$ nodes and $L_{j}(\underline{x})$ is the $\mathrm{FE}$ basis function associated to the

$j^{\text {th }} \mathrm{FE}$ node. This node location is denoted $\underline{X}_{j}^{F E}$ and has $D$ coordinates in the physical domain. For our application, the considered FE mesh is of dimension $d=2$, which means that we only work with surface elements. Note, however, that the method easily applies in the case of a volume mesh (simple extension of Fig. 1e).

The FE structure (5) thus needs to be deformed according to the deformation of the morphing box, or more precisely according to the displacement of its control points. A first way of doing so would be to take every physical point in the FE mesh $\underline{\mathcal{M}}(\underline{x})$, to find its equivalent in the morphing box parametric domain (here, we recall that we have an identity mapping, so $\underline{\xi}=\underline{\mathcal{M}}(\underline{x})$ directly), and to compute the B-spline functions at these points:

$$
\underline{\mathcal{S}} \circ \underline{\mathcal{M}}(\underline{x})=\sum_{i=1}^{n_{F F D}} N_{i}(\underline{\mathcal{M}}(\underline{x})) \underline{P}_{i},
$$

and thus for any other field of interest $\underline{\mathcal{U}}(\underline{x})$ living in the structure:

$$
\underline{\mathcal{U}}(\underline{x})=\sum_{i=1}^{n_{F F D}} N_{i}(\underline{\mathcal{M}}(\underline{x})) \underline{\tilde{U}}_{i},
$$

with $\underline{\tilde{U}}_{i}$ the value of the field of interest at the $i^{\text {th }}$ control point of the morphing box. This approach is very similar to the standard practice in the fictitious domain community where a mechanical domain with complex geometry is not meshed but embedded in a grid used for field representation, while the immersed geometry is accurately captured by means of specific quadrature rules for cut grid elements (see, e.g., [57, 43, 65, 25]) However, for our purposes, this method appears to be complex (sophisticated quadrature rules, specific treatment to 
counterbalance the ill-conditioning of the resulting system), and, more importantly, is highly invasive w.r.t. standard FE so we decided to use another approach.

The aim of our work concerning data assimilation is to keep for the field description a FE mesh coming from the analysis so that a direct comparison between the measured field and a FE simulated field is possible. To this end, we apply the morphing box deformation (i.e. composition (6)) only to the nodes of the FE mesh (i.e. pointwise):

$$
\underline{\mathcal{M}}(\underline{x})=\sum_{j=1}^{n_{F E}} L_{j}(\underline{x}) \sum_{i=1}^{n_{F F D}} N_{i}\left(\underline{\xi}_{j}^{F E}\right) \underline{P}_{i}
$$

that we can write in a matrix form for each component in each dimension (using notations of Eq. (2)):

$$
\mathcal{M}_{k}(\underline{x})=\underline{L(\underline{x})^{T}} \underline{\underline{C}}_{F F D}^{T} \underline{P_{k}},
$$

where $\underline{\underline{C}}_{F F D}$ is a $\left(n_{F F D} \times n_{F E}\right)$ matrix such that $\left[\underline{C}_{F F D}\right]_{i j}=N_{i}\left(\underline{\xi}_{j}^{F E}\right)$, and $\left(\underline{\xi}_{j}^{F E}\right)_{j=1 . . n_{F E}}$ is equal to the initial position of the FE nodes (as a consequence of the identity mapping).

The same applies to any field of interest $\underline{\mathcal{U}}(\underline{x})=\sum_{j=1}^{n_{F E}} L_{j}(\underline{x}) \underline{U}_{j}$, where $\underline{U}_{j}$ is the value of the field at the $j^{\text {th }} \mathrm{FE}$ node. Indeed, since this field is defined at the nodes of the FE mesh, the FE nodes value can be controlled by the control variables $\left(\underline{\tilde{U}}_{i}\right)_{i=1 . . n_{F F D}}$ attached to the morphing box, similarly to (8):

$$
\underline{\mathcal{U}}(\underline{x})=\sum_{j=1}^{n_{F E}} L_{j}(\underline{x}) \sum_{i=1}^{n_{F F D}} N_{i}\left(\underline{\xi}_{j}^{F E}\right) \underline{\tilde{U}}_{i} .
$$

For each component in each dimension, the above relation reads in matrix form:

$$
\mathcal{U}_{k}(\underline{x})=\underline{L(\underline{x})^{T}} \underline{\underline{C}}_{F F D}^{T} \underline{\tilde{U}_{k}}
$$

It results in a conventional FE field, but where the dof are controlled by the morphing box. This approach can thus be seen as a kind of discretization of the (pure) fictitious domain approach (6)-(7). Unlike the latter, it has the strong interest of being non-invasive w.r.t. a standard FE code in the sense that classic FE quadrature rules can be applied and the element connectivities remain the same. This also enables to straightforwardly reconstruct 
the mesh after deformation (see again Fig. 1 for illustration). The non-invasive aspect of the method will be further accounted for in next section. Note finally that if the FE mesh is infinitely fine, the present approach is equivalent to the fictitious domain approach.

At this point, we can see that this work is a generalization of what has been proposed in [13]. Indeed, it encompasses the approach in [13] and offers more possibilities. As pointed out in [8], in general, a CAD patch can be seen as a morphing box. Indeed, Eq. (8) can read:

$$
\underline{\mathcal{M}}(\underline{x})=\sum_{i=1}^{n_{F F D}}\left(\sum_{j=1}^{n_{F E}} L_{j}(\underline{x}) N_{i}\left(\underline{\xi}_{j}^{F E}\right)\right) \underline{P}_{i} .
$$

In the case where the morphing box fits to the CAD geometry (without it being necessarily a rectangle, and in the general case where the identity mapping is not ensured), and when lower-regular lines created by knots coincide with FE element edges, a B-spline function can be expressed as a linear combination of Lagrange polynomials of same degree $([66,73])$ as follows:

$$
N_{i}(\underline{x})=\sum_{j=1}^{n_{F E}} L_{j}(\underline{x}) N_{i}\left(\underline{\xi}_{j}^{F E}\right), \quad \forall i \in\left\{1, \ldots, n_{F F D}\right\}
$$

or, in matrix form,

$$
\underline{N(\underline{\xi})}=\underline{\underline{C}}_{F F D} \underline{L(\underline{x})},
$$

where $\underline{\underline{C}}_{F F D}$ becomes the Lagrange extraction operator here.

The main advantage of the general FFD approach in this work is that any FE mesh can be embedded in an FFD morphing box, while in [13], the FE space needed to include the CAD space. The FE mesh can now be made of low-order elements, triangles and quadrangles, and can be refined, either globally or locally, allowing for instance the integration of small geometry features (holes, etc) and accurate representation of the solution close to these areas. All information is described by the control points, thus only the morphing box refinement level matters for problem solving.

Remark. For a geometry that is initially a plane, we choose the morphing box to be a bivariate patch $(\delta=2)$, and the geometry can then be deformed in a space of dimension $D=3$ (see Figs. 1c and 1d). The geometry thus obtained is not a plane anymore. One 


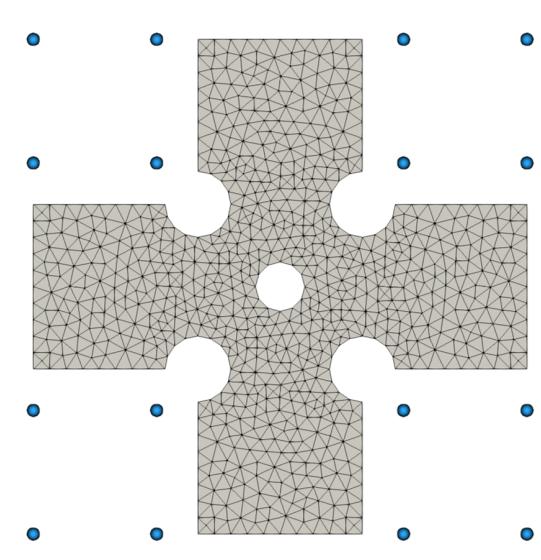

(a) Initial FE mesh and control points of the morphing box. $d=2, \delta=2, D=2$.

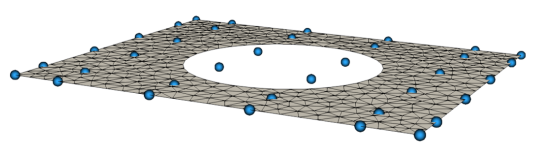

(c) Initial FE mesh and control points of the morphing box. $d=2, \delta=2, D=3$.

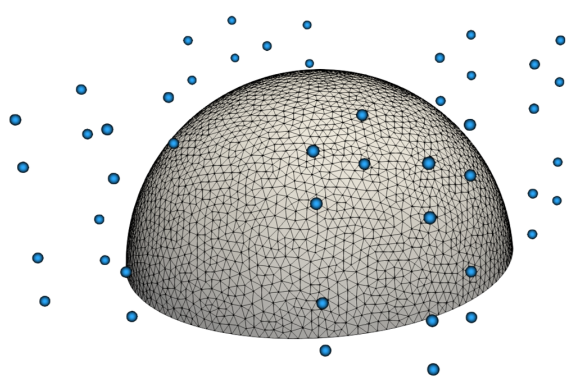

(e) Initial FE mesh and control points of the morphing box. $d=2, \delta=3, D=3$.

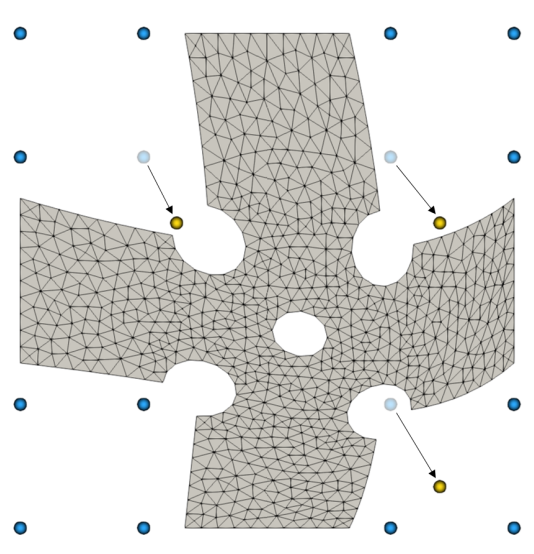

(b) Deformed FE mesh resulting from some control points displacement.

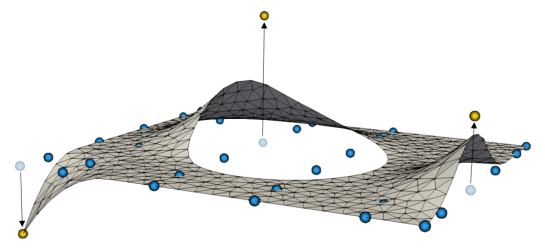

(d) Deformed FE mesh resulting from some control points displacement.

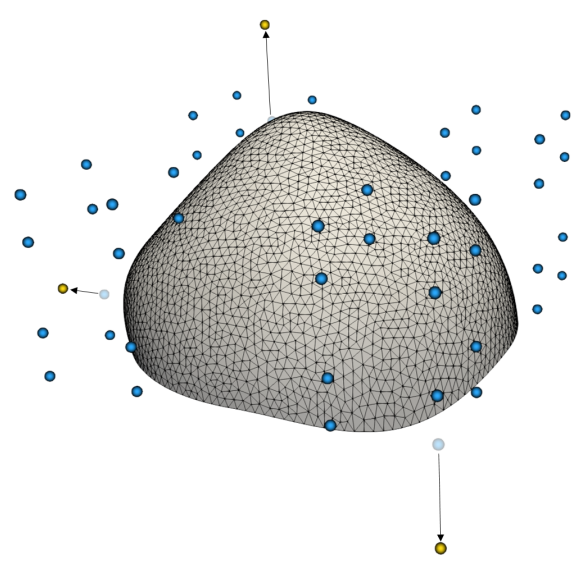

(f) Deformed FE mesh resulting from some control points displacement.

Figure 1: Deformation of a FE mesh using FFD. The blue dots are the control points of the morphing box. Moving these points (yellow dots, images on the right) results in a displacement of the FE mesh nodes. $d$ is the topology of the FE elements (1: wire, 2: surface, 3: volume), $\delta$ is the dimension of the patch for the morphing box parametric domain, and $D$ is the1gimension of the physical domain. Note that the generalization to $d=3, \delta=3$ and $D=3$ is straightforward (in this case, the surface hemisphere at the bottom just has to be viewed as a volume hemisphere). 
could wonder whether a non-planar surface FE mesh, which could however be described using B-splines, could be embedded in a bivariate patch $(\delta=2)$ with a 3D mapping. If the B-spline description is known, it is possible to do so but that means the mapping is no longer the identity function (see section 2.2). In our work, in this case, we will rather create a trivariate patch $(\delta=3)$ morphing box (see Figs. 1e and 1f).

\subsection{Implementation: projection over the morphing box}

The implementation of the FFD method mainly consists in implementing the matrix $\underline{\underline{C}}_{F F D}$ as described in (9). As we have seen, this matrix makes the link between the FE nodes position and the morphing box control points position (for each component $k \in\{1, . ., D\}$ ), such that $\underline{X}_{k}^{F E}=\underline{C}_{F F D}^{T} \underline{P_{k}}$, or for any field defined at the FE nodes, $\underline{U_{k}}=\underline{C_{F F D}^{T}} \underline{\tilde{U}_{k}}$.

The $\left(n_{F E} \times n_{F F D}\right) \underline{\underline{C}}_{F F D}^{T}$ matrix can be seen as a collection of modes, each mode being a column of the matrix [39]. For instance, if the field of interest is a displacement field, each of these modes is the displacement of all FE nodes caused by a unitary displacement of a control point. This collection of modes is a basis for a vector subspace of the vector space generated by the FE functions. We use this basis for model reduction in sections 3 and 4 where equations of the following form, emanating from the FE discretization of variational formulations, appear:

$$
\text { Find } \underline{U} \in \mathbb{R}^{D n_{F E}} \text { such that } \underline{\underline{H}} \underline{U}=\underline{B} \text {, }
$$

where $\underline{\underline{H}}$ comes from a positive symmetric bilinear form and $\underline{B}$ comes from a linear form. $\underline{U}$ will either gather FE displacement or FE shape correction dof in this article.

Let $\underline{\underline{C}}_{F F D}^{t o t}$ be the $\left(D n_{F F D} \times D n_{F E}\right)$ matrix that links all components of $\underline{U}$ (vector of size $D n_{F E}$ ) and $\underline{\tilde{U}}$ (vector of size $D n_{F F D}$ ). It can be easily computed with $\underline{\underline{C}}_{F F D}$ and a kronecker product, and it satisfies:

$$
\underline{U}=\underline{C_{F F D}^{t o t}} \underline{\tilde{U}}
$$

In accordance with the Ritz-Galerkin method, the reduced-order problem thus writes:

$$
\text { Find } \underline{\tilde{U}} \in \mathbb{R}^{D n_{F F D}} \text { such that } \quad \underline{\underline{C}}_{F F D}^{t o t} \underline{\underline{H}} \underline{\underline{C}}_{F F D}^{t o t} \underline{\tilde{U}}=\underline{\underline{C}}_{F F D}^{t o t} \underline{B} \text {. }
$$


$\underline{\tilde{U}}$ is then back converted in terms of FE dof using (16). At this stage, the non-invasiveness feature of the method clearly appears: it does not require any modifications in how $\underline{\underline{H}}$ and $\underline{B}$ are computed from a standard FE code. Similarly to [13], the developed method consists of nothing more than projecting the (expected ill-posed) FE problem onto a more regular vector subspace made of spline functions. In this sense the proposed approach may also be related to other current reduced order techniques encountered in the broad field of DIC (which will constitute our application, see sections 3 and 4). In particular, one can mention [58] where Williams' modes are used to predict crack propagation, and [19] where free vibrations modes are used to quantify the shape defects of machined surfaces. Here, we enforce less pronounced a priori knowledge so as to be able to regularize a large range of FE optimization problems: we only prescribe some regularity of the reduced basis by resorting to the family of B-spline functions. An associated interest is that it leads to a projection matrix that is very sparse. Indeed, B-spline functions have local supports and for a patch of parametric dimension $\delta$ and of degree $p$, the maximum number of non-zero functions at a given point $\underline{\xi}$ is $(p+1)^{\delta}$. It means that for each column of $\underline{\underline{C}}_{F F D}$, only $(p+1)^{\delta}$ values or less are non-zero values.

\subsection{Conditioning concerns}

As in any immersed-like method, it may be necessary to make some corrections before getting a proper basis into $\underline{\underline{C}}_{F F D}$. Indeed, in some cases, especially when the morphing box is very finely discretized, some control points may not have an influence on the embedded FE mesh, because no FE node intersects its associated B-spline support. In other words, if $\forall j, N_{i}\left(\underline{\xi}_{j}^{F E}\right)=0$, then $\underline{P}_{i}$ is non-influential (see Fig. 2). The basis is therefore corrected by removing the columns that are full of zeros in $\underline{\underline{C}}_{F F D}^{T}$, i.e. removing the non-influential control points. The obtained basis might still not be optimal, since some control points can have very little influence on the FE mesh, even though it is not strictly zero. It has consequences

on the $\underline{\underline{C}}_{F F D}^{t o t} \underline{\underline{H}} \underline{\underline{C}}_{F F D}^{t o t}{ }_{F F}^{T}$ condition number as will be seen in section 4.5. For that reason, we decided to use a criterion on the influence of a control point (through its corresponding function) derived from criteria that are used by the fictitious domain community $[65,74,60]$. 


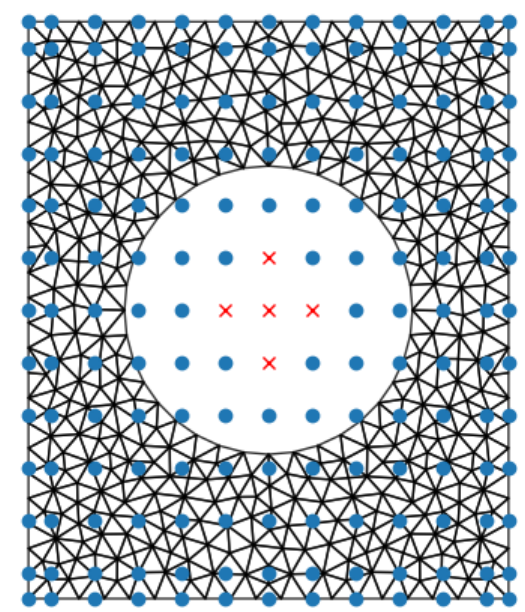

- Influential control points

$\times$ Non-influential control points

Figure 2: Non-influential points in a 2D structure with a hole.

FE structure of size $60 \times 50$ with hole of diameter 28. Nodes: 503. Elements: 884. Element type: T3.

Morphing box degree: 2, 2. Morphing box elements: $11 \times 11$.

The idea is to see what proportion of a function support actually overlaps with the embedded structure. When the structure and the morphing box are of same dimension $(d=\delta)$, we propose the following criterion for a control point $\underline{P}_{i}$ :

$$
Q_{i}=\frac{\int_{\mathcal{M}} N_{i}(\underline{\xi}) d \underline{\xi}}{\int_{I} N_{i}(\underline{\xi}) d \underline{\xi}},
$$

where $Q_{i}$ is the influence of the control point $\underline{P}_{i}$. However, when the structure and the morphing box are of different dimensions $(d \neq \delta)$, as in section 4.5 and Figs. 1c and 1d where $d=2$ and $\delta=3$, we need to define an equivalent surface from the integral along the volume, by raising it to the power $d / \delta$. Our criterion is thus enhanced as follows:

$$
Q_{i}=\frac{\int_{\mathcal{M}} N_{i}(\underline{\xi}) d \underline{\xi}}{\left(\int_{I} N_{i}(\underline{\xi}) d \underline{\xi}\right)^{d / \delta}} .
$$

This criterion could be directly coupled with the choice of a threshold to remove control points that do not have enough influence on the FE geometry. In section 4.5, we chose to 
use this criterion to only sort the points by increasing influence and then we removed the minimum of points so as to lower the $\underline{\underline{C}}_{F F D}^{t o t} \underline{\underline{H}} \underline{\underline{C}}_{F F D}^{\text {tot }} T$ condition number below a chosen value. Finally, note that for the sake of simplicity, we numerically compute $Q_{i}$ as:

$$
Q_{i}=\frac{\sum_{j} N_{i}\left(\underline{\xi_{j}}\right)}{\left(\int_{I} N_{i}(\underline{\xi}) d \underline{\xi}\right)^{d / \delta}} .
$$

The numerator is not exactly an integral (at least a mean element size is needed to get an integral approximation), but with FE elements sizes that are comparable with one another, as is the case in this article, this criterion allows to compare control points influences. The interest is that the numerator is also easily accessible because it is the sum of coefficients in the $i^{\text {th }}$ line of $\underline{\underline{C}}_{F F D}$. This sum is zero for non-influential control points, as explained above. Moreover, considering the value of B-spline functions $N_{i}$ at the nodes of the FE mesh makes sense, because they are the only points of the mesh that exactly follow the deformation of the morphing box. For instance, in the case where $\delta=2$ and $D=3$ (see Figs. 1c and 1d), Gauss points are not a priori in the morphing box, due to tessellation. To the best of our knowledge, this is the first time that such a treatment is applied in the general field of FFD.

\section{Digital Image Correlation (DIC)}

As a first application of our FFD approach, we investigate the measurement of FE displacement fields carried out by Digital Image Correlation (DIC). As mentioned in the introduction, spline functions have shown their potential for regularization in the broad field of image registration (see, e.g., [33, 68] related to the biomedical area and $[18,38,15]$ in the experimental solid mechanics community). Unlike these works, we aim here at taking advantage of spline properties, while obtaining as an output a FE displacement field which deeply simplifies the communication with a simulation software in view of performing data assimilation. A first approach in this direction was proposed in [13]. Here we extend this work to any FE mesh, regardless of the element size or type. The developed technique is particularly adapted for mechanical solutions over complex geometries that necessitate very fine FE meshes, thereby making the corresponding inverse DIC problems highly ill-posed. 


\subsection{Mesh-based DIC}

Measuring a displacement field with DIC requires taking pictures of a specimen before and after deformation. The specimen is covered with a black and white speckle in order to correlate the images of the deformed and reference state. More precisely, we seek to measure at the surface of the specimen the displacement field $\underline{u}(\underline{x})$ related to any pixel position $\underline{x}$ of the region of interest $\Omega_{s}$ in the reference image. The gray level conservation at each pixel states [30]:

$$
\forall \underline{x} \in \Omega_{s}, \quad f(\underline{x})=g(\underline{x}+\underline{u}(\underline{x})),
$$

with $f$ the reference stage image and $g$ the deformed state image. This equality cannot generally be satisfied (due to noise, brightness, speckle choice, gray level quantization...). Thus the problem is classically reformulated as the minimization of a least-square functional:

$$
\underline{u}^{\star}=\underset{\underline{u} \in\left(L_{2}\left(\Omega_{s}\right)\right)^{D}}{\arg \min } F_{u}(\underline{u}(\underline{x})) \quad \text { with } F_{u}(\underline{u}(\underline{x}))=\frac{1}{2} \int_{\Omega_{s}}[f(\underline{x})-g(\underline{x}+\underline{u}(\underline{x}))]^{2} d \underline{x} .
$$

The problem can be discretized using a FE basis (leading to so-called FE-DIC [69, 6, 20, $77,52]$ ), which means that the displacement field is constructed thanks to standard nodal Lagrange polynomial functions: $\underline{u}(\underline{x})=\underline{\underline{L(\underline{x})^{T}}} \underline{\underline{U}}$. In this expression, $\underline{\underline{L(\underline{x})}}$ is written as a matrix to handle all physical dimensions at once. In the case of $2 \mathrm{D}-\mathrm{DIC}$, we have $D=d=2$ (see Figs. 1a and 1b). Note however that the developed FFD-based methodology could be straightforwardly applied to DVC [41, 24]. These functions are linked to a FE mesh that we suppose to have a priori. It usually comes from FE simulations to then faster the dialog between measured and simulated fields for data assimulation in experimental mechanics $[54,59,47]$. We recall that the proposed FFD approach does not impose any constraint on the FE mesh construction (see section 2).

The FE-DIC problem writes:

$$
\underline{U}^{\star}=\underset{\underline{U} \in \mathbb{R}^{D n_{F E}}}{\arg \min } F_{u}\left(\underline{\underline{L(\underline{x})^{T}}} \underline{\underline{U}}\right)
$$


This problem is solved using a modified Gauss-Newton algorithm [53]. An initialization of the displacement $\underline{U}^{0}$ at each dof is chosen (see discussion at the end of the section), and at each iteration $k$, a correction $\underline{\delta U^{k}}$ is sought so that $\underline{U}^{k}=\underline{U}^{k-1}+\underline{\delta U^{k}}$. The descent direction $\underline{\delta U^{k}}$ is the solution of the following linear system:

$$
\underline{\underline{H_{u}}} \underline{\delta U^{k}}=\underline{B_{u}}{ }^{k} \quad \text { with }\left\{\begin{array}{l}
\underline{\underline{H_{u}}}=\int_{\Omega_{s}} \underline{\underline{L(\underline{x})}} \underline{\nabla f}(\underline{x}) \underline{\nabla f}(\underline{x})^{T} \underline{\underline{L(\underline{x})^{T}}} d \underline{x} \\
\underline{B_{u}{ }^{k}}=\int_{\Omega_{s}} \underline{\underline{L(\underline{x})}} \underline{\nabla f}(\underline{x})\left[f(\underline{x})-g\left(\underline{x}+\underline{\underline{L(\underline{x})^{T}}} \underline{U}^{k-1}\right)\right] d \underline{x}
\end{array},\right.
$$

where $\underline{\nabla f}(\underline{x})$ is the gradient of image $f$ evaluated at position $\underline{x}$. A brightness correction is applied at the element level so that the graylevel values in each element have the same mean and standard deviation [13]. In case of a too fine mesh compared to the data provided by the experimental instrumentation, the FE-DIC problem (23) is highly ill-posed. As a strong regularization, we apply the FFD projection strategy described in section 2. More precisely, we seek the displacement field in the reduced basis coming from a morphing box; that is, we express the unknown field as in (11) which leads to modify, at each iteration, system (24) by the one given in (17).

For the initialization, we use a multiscale approach, inspired from [13], which combines coarse graining techniques used in DIC and multilevel properties of splines. Coarse graining $[61,56]$ is a well-known tool in DIC to avoid local minima when solving the problem. It consists in aggregating pixels in the images $f$ and $g$ to create images with lower resolution, and consider them to run the DIC algorithm first. The solution is then used as an initialization of the DIC problem on the well-resolved images. This process can be repeated several times (see Fig. 3). In our approach, since splines can be refined automatically and efficiently, it is interesting to couple the morphing box refinement with the coarse graining procedure. Indeed, when the images resolution is low, we need a spline box with few elements to regularize the corresponding coarse DIC problem. More precisely, at each scale $i$, the final coarse spline dof $\underline{\tilde{U}}_{\text {scale } i}^{n}$ is back converted in terms of FE dof $\underline{U}_{\text {scale } i}^{n}$ following (16), and then taken as the initialisation for the finer scale $i-1$ : $\underline{U}_{\text {scale } i}^{n}=\underline{U}_{\text {scale } i-1}^{0}$ (see Fig. 3).

The multilevel properties of B-splines avoid computing the FFD matrix at each scale. Only the computation of $\underline{\underline{C}}_{F F D_{\text {scale } 0}}^{\text {tot }}$ at the finest scale, namely scale 0 , as described in 
section 2.3, is needed. Then, from Eq. (4) applied to the spline dof of interest $\underline{\tilde{U}}$, we get that $\underline{\tilde{U}}_{\text {scale } i}=\underline{\underline{C}}_{\text {ref } i, i+1}^{\text {tot }} \underline{\tilde{U}}_{\text {scale } i+1}$. At a given scale $s$, the FFD matrix thus writes:

$$
\underline{\underline{C}}_{F F D_{\text {scale s }}}^{t o t}=\underline{\underline{C}}_{\text {ref } s-1, s}^{t o t} \cdots \underline{\underline{C}}_{r e f 1,2}^{t o t} \underline{\underline{C}}_{\text {ref } 0,1}^{\text {tot }} \underline{\underline{C}}_{F F D_{\text {scale } 0}}^{t o t} .
$$

In the end, the resolution of the regularized DIC problem at each scale simply consists in modifying system (24) by the one given in (17) with $\underline{\underline{C}}_{F F D}^{t o t}$ replaced by $\underline{\underline{C}}_{F F D_{\text {scale }}}^{\text {tot }}$.

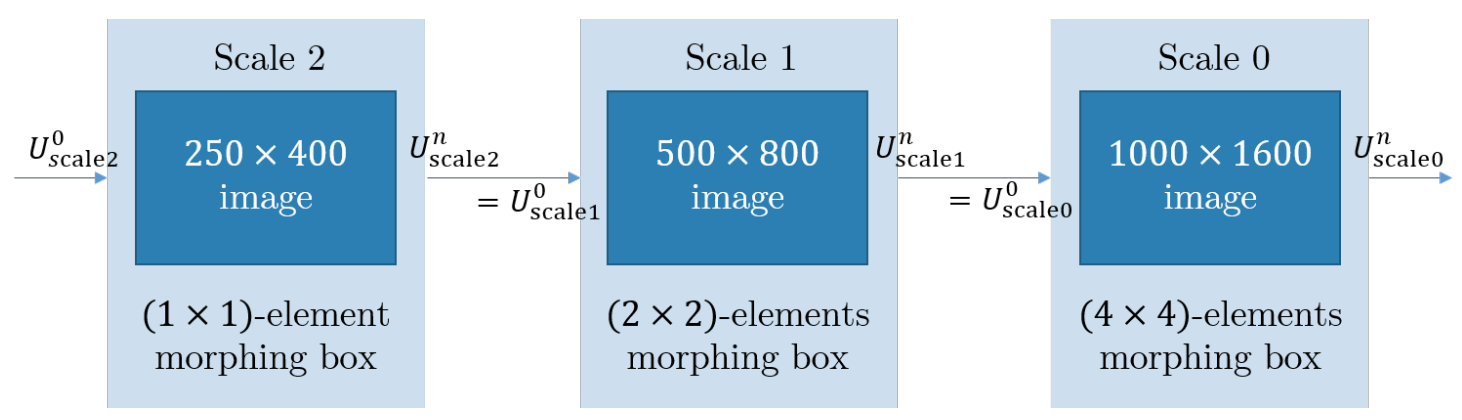

Figure 3: Example of a multiscale approach. At scale 0, the images $f$ and $g$ are used with their initial resolution, and a number of spline elements is chosen for the FFD morphing box. For each scale, the pixels in $f$ and $g$ are aggregated by $2^{2 \times \text { scale }}$, and similarly, the number of spline elements is reduced to achieve a sufficient regularization level. The number of scales can be chosen freely, but the images must still contain enough graylevel gradient.

\subsection{D digital image correlation: Bending of a beam}

In order to validate our method for mesh-based DIC, we applied it on a bending beam. A 2D simply supported horizontal aluminium beam receives vertical loads on two points (see Fig. 4a). The displacement field is then measured on a $1006 \times 5500$-pixel region of interest. The chosen FE mesh is a structured quadrangular mesh $(20 \times 110$ 4-node elements $)$ but could be any mesh. Each FE node can move along the two planar directions. A 3-scale initialization is chosen, which means that the pixels of images $f$ and $g$ are aggregated 8 by 8 at the coarsest scale. This initialisation was carried out using FFD regularization with a single 2 -dimensional quadratic patch of $1 \times 1,2 \times 2$ and $4 \times 4$ spline elements at scale 3,2 and 1 respectively. 
The deformed configuration eventually obtained at scale 0 is shown in Fig. 4b. The final displacement field was computed with the proposed FFD regularization technique with $8 \times 8$ spline elements. We compared our results with two classical solutions obtained with the same initialization, only changing the way of solving the problem at scale 0: (i) what is commonly called Tikhonov regularization in the field of DIC $[50,16,56]$ and (ii) no regularization. Tikhonov regularization consists here in a penalization of the $L_{2}$-norm of the gradient of each component of the displacement field. In this case, the penalization coefficient was chosen so as to obtain a characteristic regularization length comparable to the smallest regularization length that we get with the FFD regularization at scale 0. The smallest FFD regularization length is set along $y$ because the FFD spline elements are smaller in that direction. This choice comes from the fact that the variations of the sought displacement field are known to be greater along $y$. The FFD characteristic regularization length in that direction is thus 2 times the size of the spline elements in the $y$ direction. In order to set the Tikhonov penalization coefficient, the procedure described in [41] was followed, with the choice of a reference displacement field in the form of a plane wave. Note here that defining a physical regularization length is straightforward with the FFD method, whereas additional computations are required for its estimation with the Tikhonov regularization.

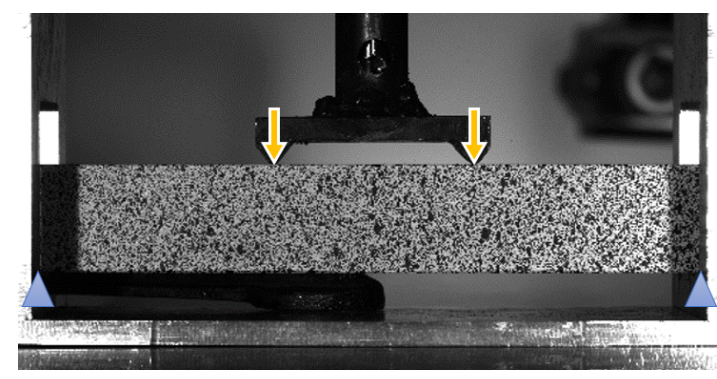

(a) Experimental set-up. Yellow arrows and blue triangles represent respectively applied loads in the $\underline{y}$ direction and points where the beam is simply supported.

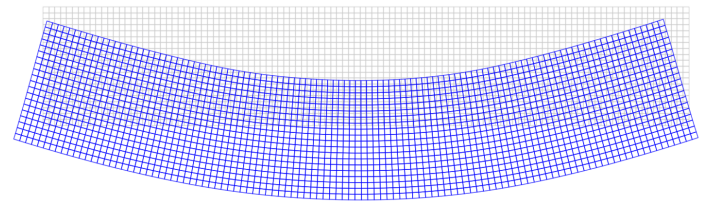

(b) Gray and blue meshes represent respectively the undeformed FE mesh and its deformed configuration after running DIC with our FFD regularization. Amplification factor: $\times 30$.

Figure 4: Experimental application: bending beam.

The FFD regularization shows great potential for reducing the noise impact on the result. 


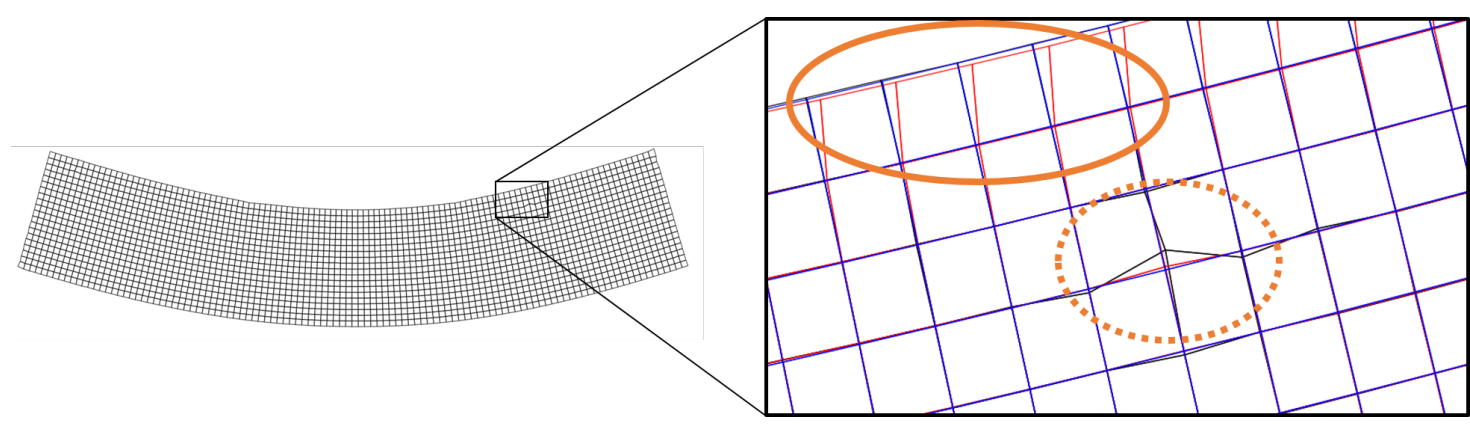

Figure 5: Zoom on defects that are not part of the displacement field. The black mesh results from the unregularized problem, the red mesh is obtained using Tikhonov regularization, and the blue mesh shows the deformed configuration with FFD regularization. The dotted circle shows the impact on the results of dust on the camera, which affects the black and red meshes. The solid circle shows artefacts inherent to the standard Tikhonov regularization.

The obtained displacement field with FFD regularization is consistent and very smooth, as shown in Fig. 5. Two harmful effects can be reported with the standard alternatives to solve the problem (see the region within the dotted and solid circles in Fig. 5). The dotted circle indicates the impact of dust on the camera. A speck of dust creates a small black spot in the images, and that spot remains stationary relatively to the images. Thus it does not follow the displacement of the specimen. This explains why a node of the FE mesh has a smaller displacement than its neighbors when no regularization is applied (black mesh). Tikhonov regularization (red mesh) smooths this impact, and the chosen FFD regularization (blue mesh) completely removes the problem. The solid circle shows Tikhonov defects on the curved edges of the specimen. Indeed, it reduces a part of the rotations that is relevant, which creates artefacts on the edges. This harmful effect of the standard Tikhonov regularization has already been underlined in [13] for the specific case of mesh-based shape measurement.

The differences between FFD regularization, Tikhonov regularization and no regularization are all more noticeable when displaying the strain field, since the impact of noise tends to be increased when taking a derivative. Fig. 6 shows the strain fields obtained with no regularization, Tikhonov regularization, FFD regularization and an idealized analytical solution obtained with a standard beam model and simple boundary conditions. Clearly, using no regularization gives noisy results (see Figs. 6a to 6c). Color scales have been adjusted in 


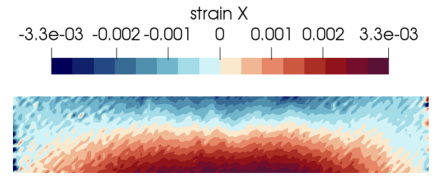

(a) No regularization, $\varepsilon_{x x}$

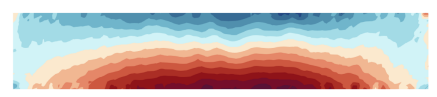

(d) Tikhonov regularization, $\varepsilon_{x x}$

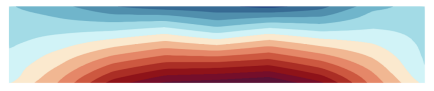

(g) FFD regularization, $\varepsilon_{x x}$

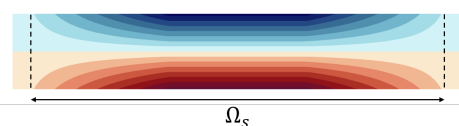

(j) Analytical solution, $\varepsilon_{x x}$

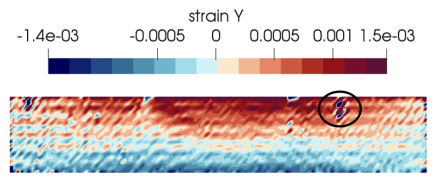

(b) No regularization, $\varepsilon_{y y}$

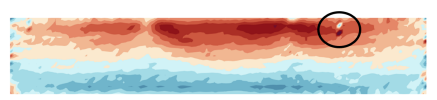

(e) Tikhonov regularization, $\varepsilon_{y y}$

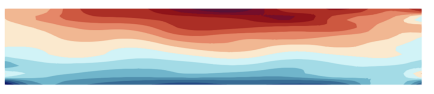

(h) FFD regularization, $\varepsilon_{y y}$

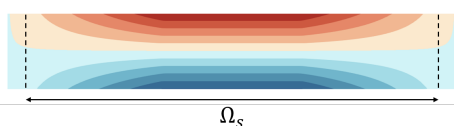

(k) Analytical solution, $\varepsilon_{y y}$

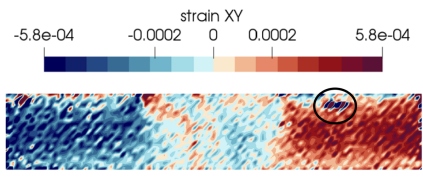

(c) No regularization, $\varepsilon_{x y}$

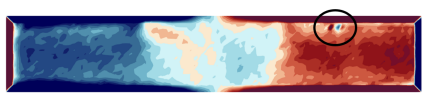

(f) Tikhonov regularization, $\varepsilon_{x y}$

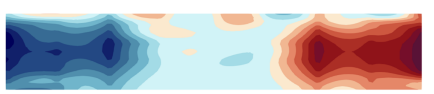

(i) FFD regularization, $\varepsilon_{x y}$

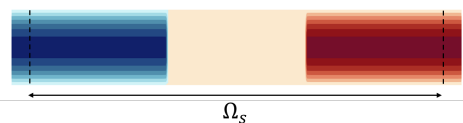

(1) Analytical solution, $\varepsilon_{x y}$

Figure 6: Comparison of our regularization method (third line, FFD regularization) with the results obtained with no regularization (first line), with Tikhonov regularization (second line) and with an idealized beam solution (fourth line) for the measured strain coming from DIC on the bending beam shown at figure $4 \mathrm{a}$. The beam for the analytical solution is longer because it represents the whole beam while the other figures only represent the region of interest. The impact of dust has been circled.

order to see the relevant components of the strain field. The edges of the region of interest and the region where there is the dust spot are undergoing unrealistic off-scale values. For example, the top right corner of the region of interest has values of $\varepsilon_{y y}$ up to $1.3 e-2$, that is 10 times higher than expected. Tikhonov regularization reduces the impact of the noise, makes the edges much less erratic and smooths the impact of dust on the result. However, as pointed out in Fig. 5, the rotations of the edges are dampened, and that leads to measuring some spurious shear (see near to the top and bottom boundaries of the region of interest in Fig. 6f in comparison to the reference in Fig. 61). The FFD regularization gives smooth results thanks to the projection of the DIC problem onto a smaller, more regular, spline basis. It gives satisfying results compared to the idealized analytical solution. This appears totally relevant since the aim of the experiment was to measure a global displacement field, in accordance with Saint Venant's principle. 
Remark. Here we used FFD regularization for the initialization in each case (process illustrated in Fig. 3). Note that in case another regularization technique is chosen to compute an initialization $\underline{U}_{\text {scale } 0}^{0}$ (such as Tikhonov regularization as is often done in the field $[50,16,56]$ ), a projection is needed before starting the developed FFD-DIC resolution process at scale 0 . Indeed, when performing an optimization, it is essential to start with the initialization in the research space. In other words, we have to make sure that the initialization performed is not adding features that cannot be modified in the FFD subspace. In this case, the projection of

$\underline{U}_{\text {scale } 1}^{n}$ consists in finding $\underline{U}_{\text {scale0 }}^{0}$ that minimizes the $L_{2}$-error $\left\|\underline{U}_{\text {scale1 }}^{n}-\underline{C}_{F F D}^{t o t} \underline{U}_{\text {scale0 }}^{T}\right\|^{2}$. The resulting FE field used for initialization is finally $\underline{U}_{\text {scale0 }}^{0}=\underline{\underline{C}}_{F F D}^{\text {tot }} \underline{\underline{U}}_{\text {scale0 }}^{0}$.

\section{Shape measurement}

Spline-based regularization is particularly well adapted when the measured field is expected to be smooth [16]. It is the case when measuring a shape before performing 3D displacement field measurement. This shape measurement procedure is indispensable before performing any experiment.

\subsection{Mesh-based shape measurement}

Mesh-based shape measurement consists in correcting the theoretical geometry of a structure using its FE mesh by analyzing several pictures of the structure taken from different points of view. These corrections are needed because the real geometry may slightly differ from the nominal geometry, due to machining imperfections or to the experimental set-up that can induce small deformations. This mismatch between the idealized CAD and the actual specimen shape may be detrimental to the displacement measurement.

Without lack of generality, we consider two images $f_{0}$ and $f_{1}$ given by two cameras. Each camera is modelled by a mapping $\underline{\Pi}_{c}$ from the physical space $\mathbb{R}^{3}$ to the image in pixels [71]:

$$
\begin{aligned}
\underline{\Pi}_{c}: \Omega_{s} \subset \mathbb{R}^{3} & \longrightarrow \mathbb{R}^{2} \\
\underline{x} & \longmapsto \underline{x_{c}}=\underline{\Pi}_{c}(\underline{x})
\end{aligned}
$$

Such models depend on intrinsic parameters (such as focal length, image center, horizontal/vertical aspect ratio, skew and distortion parameters), determined via a photogram- 
metric calibration phase using targets [22], and extrinsic parameters (camera position and orientation in the physical space), found during a first calibration procedure that will not be detailed here.

The goal of shape measurement is to find a geometry correction field $\underline{s}(\underline{x})$ such that the projection of a physical point $\underline{x}+\underline{s}(\underline{x})$ of the structure has the same graylevel on both images. The problem thus writes:

$$
\underline{s}^{\star}=\underset{\underline{s} \in\left(L_{2}\left(\Omega_{s}\right)\right)^{D}}{\arg \min } F_{s}(\underline{s}(\underline{x})) \quad \text { with } F_{s}(\underline{s}(\underline{x}))=\int_{\Omega_{s}} r^{2}(\underline{s}(\underline{x})) d \underline{x}
$$

with $r$ the graylevel residual field:

$$
r(\underline{s}(\underline{x}))=\left[f_{0} \circ \underline{\Pi}_{0}(\underline{x}+\underline{s}(\underline{x}))-f_{1} \circ \underline{\Pi}_{1}(\underline{x}+\underline{s}(\underline{x}))\right] .
$$

As for DIC (see section 3), we discretized the problem using a FE basis and solved it with a modified Gauss-Newton algorithm. An initialization of the shape correction at each degree of freedom $\underline{S}^{0}$ is set (often at zero since the discrepancy between theoretical and actual shape is usually small) and the shape correction at each iteration, $\underline{\delta S}^{k}$ such that $\underline{S}^{k}=\underline{S}^{k-1}+\underline{\delta S}^{k}$, is computed by solving the following linear system:

$$
\begin{aligned}
& \underline{\underline{H}}_{s}^{k} \underline{S}^{k}=\underline{B}_{s}^{k} \\
& \text { with }\left\{\begin{array}{l}
\left.\underline{\underline{H}}_{s}^{k}=\int_{\Omega_{s}} \underline{\underline{L(\underline{x})}}\left(\underline{\underline{J}}_{\Pi_{0}}^{k^{T}} \underline{\nabla f_{0}}{ }^{k}-\underline{\underline{J}}_{\Pi_{1}}^{k^{T}} \underline{\nabla f_{1}}\right)\left(\underline{\underline{J}}_{\Pi_{0}}^{k^{T}} \underline{\nabla f_{0}}{ }^{k}-\underline{\underline{J}}_{\Pi_{1}}^{k^{T}} \underline{\nabla f_{1}}\right)^{T} \underline{\underline{L(\underline{x}}}\right)^{T} d \underline{x}, \\
\underline{B}_{s}^{k}=\int_{\Omega_{s}} \underline{\underline{L(\underline{x})}}\left(\underline{\underline{J}}_{\Pi_{0}}^{k^{T}} \underline{\nabla f_{0}}{ }^{k}-\underline{\underline{J}}_{\Pi_{1}}^{k} \underline{\nabla f_{1}}\right) r\left(\underline{\underline{L(\underline{x})}} \underline{S}^{k-1}\right) d \underline{x}
\end{array},\right.
\end{aligned}
$$

where $\underline{J}_{\Pi_{c}}^{k^{T}}$ is the jacobian matrix of the mapping $\underline{\Pi}_{c}$ defined at eq. (26), with respect to $\underline{x}$, calculated at the physical point $\underline{x}+\underline{\underline{L(\underline{x}}})^{T} \underline{S}^{k-1}$, and $\underline{\nabla f_{c}}{ }^{k}=\underline{\nabla f_{c}} \circ \underline{\Pi}_{c}\left(\underline{x}+\underline{\underline{L(\underline{x}}} \underline{S}^{k-1}\right)$ defines the gradient of the graylevel image.

Similarly to previous section, the matrix $\underline{\underline{H}}_{s}^{k}$ and the vector $\underline{B}_{s}^{k}$ can be computed by any pre-existing FE-SDIC software. We regularize the problem by projecting it on the smoother FFD space thanks to the matrix $\underline{\underline{C}}_{F F D}^{t o t}$ :

$$
\underline{\underline{C}}_{F F D}^{t o t} \underline{\underline{H}}_{s}^{k} \underline{\underline{C}}_{F F D}^{t o t} \underline{\delta}^{k}=\underline{\underline{C}}_{F F D}^{t o t} \underline{B}_{s}^{k}
$$


It must be stated that, with such a projection on a regular sub-space, the obtained shape correction field is required to be smooth but, depending on the initial FE mesh, the measured shape may not be regular.

The multiscale approach as described in Fig. 3 is also used for shape measurement.

\subsection{Generalization to more cameras}

In section 4.5, we measure a shape from more than two angles. In this case, each pair of cameras has to be considered. Hence, with $N_{\text {cam }}$ cameras, the problem becomes:

$$
\underline{s}^{\star}=\underset{\underline{s} \in L_{2}\left(\Omega_{s}\right)}{\arg \min } \sum_{i=1}^{N_{\text {cam }}} \sum_{j=i+1}^{N_{\text {cam }}} \int_{\Omega_{s}} V_{i}(\underline{x}) V_{j}(\underline{x}) r_{i j}^{2}(\underline{s}(\underline{x})) d \underline{x},
$$

with $V_{c}(\underline{x})$ a mask associated to camera $c$, such that $V_{c}(\underline{x})=1$ if a point on the FE model $\underline{x}$ is correctly seen by the camera $c$ and $V_{c}(\underline{x})=0$ otherwise. Indeed, with $3 \mathrm{D}$ structures, a given camera may not see the whole region of interest at once (see section 4.5). Moreover, when measuring the shape from several points of view, two cameras are very unlikely to have equal visible structure parts. For that reason, the contribution of a pair of cameras is restricted to the intersection of visible regions of each camera. $V_{i}(\underline{x}) V_{j}(\underline{x})$ is thus a mask associated to the pair of cameras $i$ and $j . r_{i j}$ is similar to $r$ defined above:

$$
r_{i j}(\underline{s}(\underline{x}))=\left[f_{i} \circ \underline{\Pi}_{i}(\underline{x}+\underline{s}(\underline{x}))-f_{j} \circ \underline{\Pi}_{j}(\underline{x}+\underline{s}(\underline{x}))\right] .
$$

Consequently, the computation of the matrix $\underline{\underline{H}}_{s}^{k}$ and the vector $\underline{B}_{s}^{k}$ involves a sum of all pairs contribution. Note that for many pairs of cameras, $V_{i}(\underline{x}) V_{j}(\underline{x})=0, \forall \underline{x}$, because both cameras see different regions of the structure. This significantly reduces the number of terms in the double sum.

Practically speaking, $V_{c}$ is defined for each FE element. Obviously, elements which outward normal unit vector $\underline{n}_{e}$ is pointing to the background are not visible. As a result, the value of $V_{c}$ is set to 0 for any $\mathrm{FE}$ elements that meet the criterion $\underline{Z}_{c} \cdot \underline{n}_{e}<0$, where $-\underline{Z}_{c}$ is the unit direction the camera $c$ is looking at.

We also did not consider surfaces that are seen too inclined by the camera, because the speckle pattern is not accurately captured due to a too small pixel size to structure surface 
ratio. When this criterion is below a given threshold $\epsilon_{n}$, the element $e$ is considered not correctly visible for the camera $c$, and $V_{c}(\underline{x})=0$ on this element. In other terms:

$$
V_{c}(\underline{x})= \begin{cases}1 & \text { if } \underline{Z}_{c} \cdot \underline{n}_{e}(\underline{x})>\epsilon_{n}, \\ 0 & \text { otherwise. }\end{cases}
$$

Note here that the greater we choose the threshold $\epsilon_{n}$, the more elements are 'correctly' seen by each camera, and the more cameras see a given element. The minimum number of cameras that are needed for shape measurement is thus defined by this threshold, since each FE element should be seen by at least two cameras for the FE problem to be solvable.

Note also that all elements are not seen by the same number of cameras (zones that are seen by a given camera overlap), which means some dof have more weight than others by construction of $\underline{\underline{H}}_{s}^{k}$. We decided to normalize each element contribution by the number of pairs of cameras that can see it, such that no dof is predominant in the minimization of the functional. For other purposes, it could be useful not to normalize, for example if some parts of the structure are more interesting than others and have more cameras pointing at them.

\subsection{FFD projection of the shape measurement problem}

So far shape correction consists in moving nodes (or control points) in the 3 dimensions of space. However, moving a node/control point in the direction tangent to the surface does not change the geometry and thus makes the shape measurement problem ill-posed. To avoid mesh distortion or elements overlapping and to improve the spectral properties of the Hessian, the shape correction is sought in one single direction, usually the normal to the surface [56]. As such, we end up with a scalar problem, namely, with only one dof per node. In FFD-based 3D shape optimization, the usual practice consists in proceeding in two successive steps: (i) first the 3D FE optimization problem is projected onto the 3D Spline shape functions, then (ii) the control points are constrained to move in one direction only.

Defining a direction vector field at the FFD control points is not completely satisfactory in our context because of the multiscale process. The direction field variation must be constant throughout the scales so that the final results do not depend on the minimization 

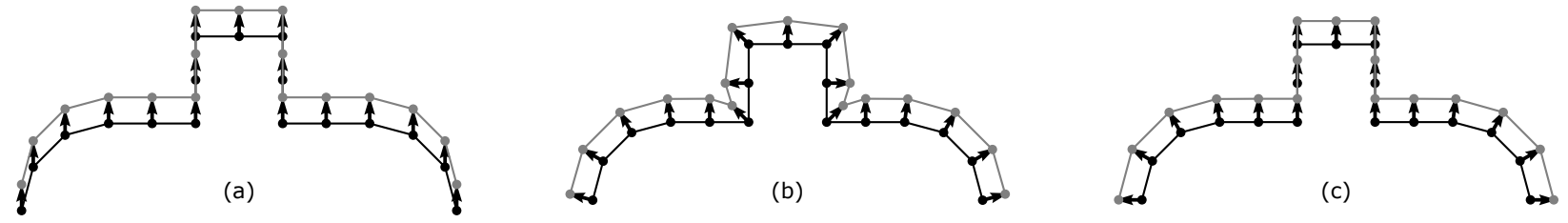

Figure 7: Examples of normal fields defined on the FE mesh.

algorithm. This condition implies that the direction field must be included in the coarsest FFD approximation sub-space. This constraint may be restrictive especially when dealing with complex geometries.

In the spirit of the approach developed from the finite element mesh point of view, we propose to define a local normal direction field $\underline{n}$ defined at the nodes of the FE mesh and along which the nodes will be moved to update the geometry. It means we look for a shape correction written $\underline{S}=\underline{\underline{R_{n}}} \underline{S_{n}}$, where $\underline{S_{n}}$ contains dofs in the chosen direction and $\underline{\underline{R_{n}}}$ is the corresponding extraction $D n_{F E} \times n_{F E}$ matrix gathering the components of unit normal vectors $\underline{n}$ at each node, $n_{F E}$ being the number of FE nodes as introduced in (5). The 3D FE optimization problem is then first projected according to this normal field so that the operator and right-hand side become $\underline{\underline{R_{n}}} \underline{\underline{H}}_{s}^{k} \underline{\underline{R_{n}}}$ and $\underline{\underline{R_{n}}}{ }^{T} \underline{B}_{s}^{k}$, respectively. Hence, the FE shape correction field becomes a scalar problem (one dof per node). Only then is this scalar problem projected onto the FFD space, which means we look for a shape correction $\underline{S_{n}}=\underline{\underline{C}}_{F F D} \underline{\tilde{S}_{n}}$. The FFD projected problem eventually writes:

$$
\underline{\underline{C}}_{F F D} \underline{\underline{R_{n}}} \underline{\underline{H^{k}}} \underline{\underline{R}}^{\underline{R_{n}}} \underline{\underline{C}}_{F F D}^{T} \underline{\delta \tilde{S}_{z}}=\underline{\underline{C}}_{F F D} \underline{\underline{R_{n}}} \underline{\underline{B}} \underline{B}_{s}^{k}
$$

The corresponding dofs $\underline{\tilde{S}_{n}}$ do no longer correspond to displacements of the control points, but to magnitudes of the correction along the FE nodes normals. This choice makes it possible to keep a normal which is constant throughout the scales but not constrained by the coarsest length scale. The method is general, since the normals can be defined in many different ways, see Fig. 7: for instance a field of local normals (nodes by nodes) (Fig. 7(b)), a field of non-local normals taking into account the geometry of the neighborhood (Fig. 7(a)) or even user-defined normals (Fig. 7(c)).

To the best of our knowledge, such an approach is original in the general field of FFD, 
since in our work the direction of the correction is defined on the FE mesh and only the magnitude of the correction field is defined by the FFD control points.

\subsection{Twisted plate with a hole}

We applied the proposed regularization method to the shape measurement of a set or real images already studied in [13]. It consists in a steel open-hole tensile specimen of size $180 \times 50 \times 2 \mathrm{~mm}$ with a 28 -mm diameter. A speckle pattern was laser printed over a layer of uniform white paint. 8 bits $2452 \times 2052$ digital images were obtained thanks to two 5 Mpx CCD cameras and $50 \mathrm{~mm}$ lenses. Cameras were positioned with a $25^{\circ}$ stereo-angle, which represents a good compromise between in-plane and out-of-plane uncertainties [2, 32]. The specimen was clamped in an electro-mechanical tensile testing machine. A misalignment of 2 or $3^{\circ}$ of the jaws twisted the specimen.

In this example, the initial FE mesh is flat. Consequently, a bivariate patch is chosen for the morphing box. Since the outer shape of the structure is a rectangle, we choose a FFD morphing box that coincides with the structure on the edges. The high resolution of our images allows to use 4 scales for the shape measurement problem resolution. We chose to start at the coarsest scale with a 1-element FFD morphing box, and then subdivide each spline element into 4 elements at each finer scale, so that the finest scale has a 64-element FFD morphing box.

The problem to solve is the one given in equation (34). In the case of an initial flat configuration, the obvious choice for $\underline{n}$ at each FE node is the normal to the surface, which is the same direction for all nodes. In this special case where all nodes can be corrected in the same direction, it is possible to see the correction as a displacement of the FFD control points along that same direction, which made the visualization of the deformed FFD morphing box possible on Fig. 8.

The results are shown Fig. 8. As expected, we observe very smooth results with the FFD regularization, because we benefit from the regularity of spline functions and the very low number of dof in the regularized problem. The coarsest scale already gives satisfying results and finer scales slightly improve this shape correction field. Results are in good agreement 


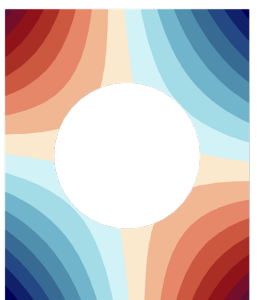

(a) $U_{z}$ field, scale 3

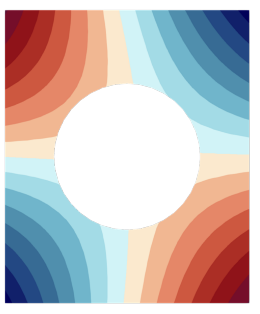

(d) $U_{z}$ field, scale 2 .

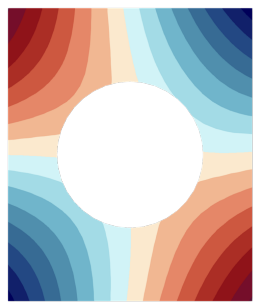

(g) $U_{z}$ field, scale 1.

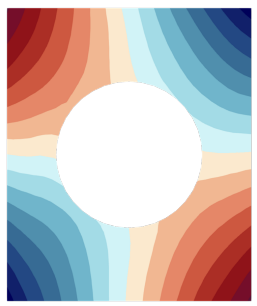

(j) $U_{z}$ field, scale 0

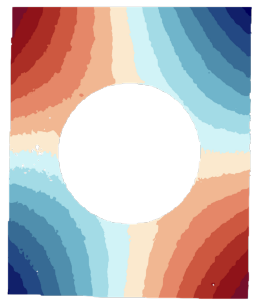

(m) $U_{z}$ field, subset-DIC.

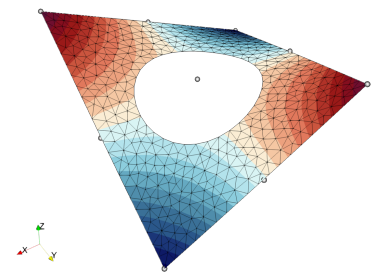

(b) FE mesh, scale 3 .

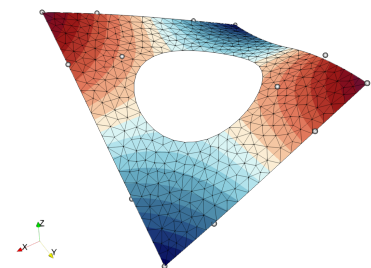

(e) FE mesh, scale 2 .

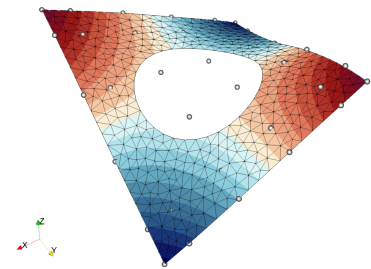

(h) FE mesh, scale 1.

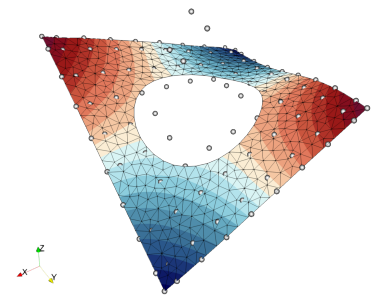

(k) FE mesh, scale 0.

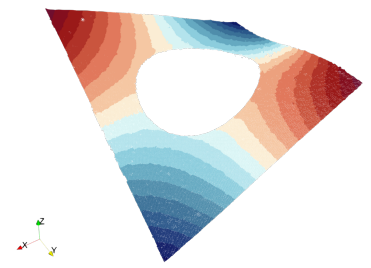

(n) Subset-DIC measurement.

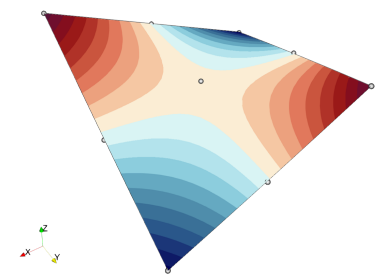

(c) FFD morphing box, scale 3 .

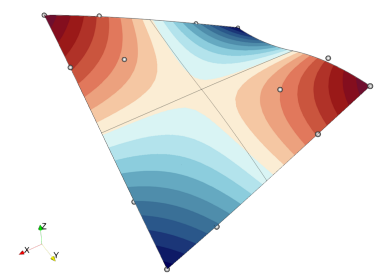

(f) FFD morphing box, scale 2.

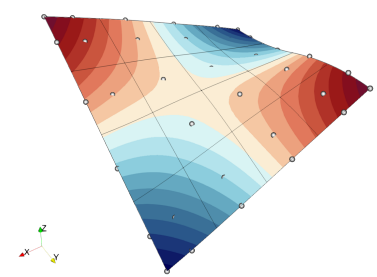

(i) FFD morphing box, scale 1 .

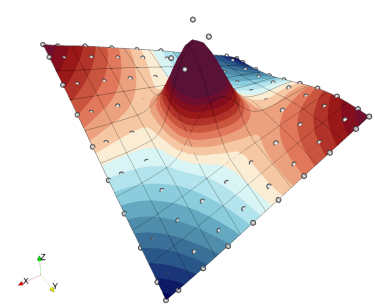

(l) FFD morphing box, scale 0 .

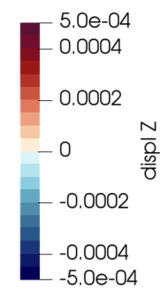

(o) Color scale: displacement in $\mathrm{mm}$.

Figure 8: Experimental application: Twisted plate with a hole. We compare the shape measured with our method using a 3-scale initialization with the shape measured thanks to the industrial software VIC. 
with the shape correction field obtained with subset DIC method. Here, VIC 3D software was used.

On the right, Fig. 8 shows the shape correction field expressed in each point of the FFD morphing box, using $2^{\text {nd }}$-degree B-spline functions. Higher values in the center can be noticed at the finest scale. This feature results from the too little influence that control points inside the center hole have on the FE mesh. It can be interpreted as a fictitious extension of the shape correction field outside the region of interest, i.e. outside the FE mesh, that inevitably exists when the mesh does not occupy the whole morphing box. It does not have a physical meaning, but it does not affect the solution if the problem is not ill-conditioned. For this example, it does not negatively impact the results.

An advantage of this method compared to a standard CAD-based method as in [13] is the absence of $C^{0}$ lines that appear when creating a boundary-fitted patch. The FFD regularization is a generalization of the CAD-based regularization proposed in [13], where we eliminated the need for an analysis-suitable boundary-fitted spline mesh. As a result, the FFD regularization can be applied to any geometry. Another advantage coming from the fact that the FE mesh does not have to derive from a spline mesh is that any type of FE element can be chosen, and not only 9-node quadrilaterals, that are not implemented in all FE softwares. The next example illustrates a case on an initially non-planar geometry.

\subsection{Spherical cap}

The proposed FFD approach offers great possibilities to regularize shape measurement on complex 3D shapes. In this section, we apply this method to a spherical cap (see Fig. 9). The initial mesh for the spherical cap is $35.45 \mathrm{~mm}$ large and $77.10 \mathrm{~mm}$ high. This FE mesh was constructed as a part of a sphere with a $38.686-\mathrm{mm}$ radius.

A set of 14 images of size $2560 \times 2048$-pixels was taken, including 11 from different sides of the spherical cap (Fig. 9a), with view points placed all over the structure, and 3 from above (Fig. 9b), with different view angles. On the images, on the "correctly visible" part of the sphere, one pixel represents approximately $0.125 \mathrm{~mm}$. Because each camera cannot correctly see the whole region of interest, we applied the mask $V_{c}$ defined in (33) with an $\epsilon_{n}$ 


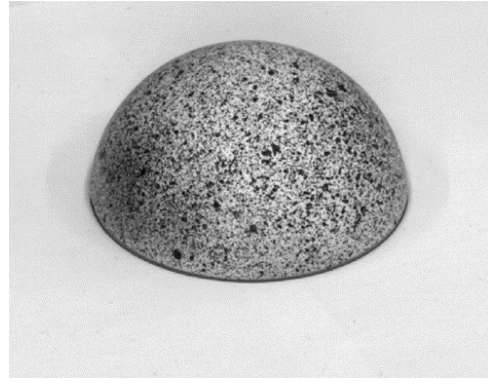

(a) Image taken from the side.

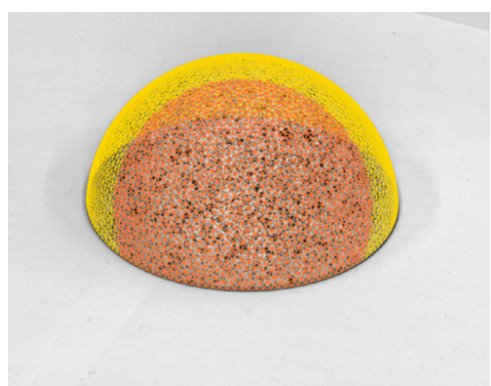

(c) In red, visible mesh for the camera that took the above picture.

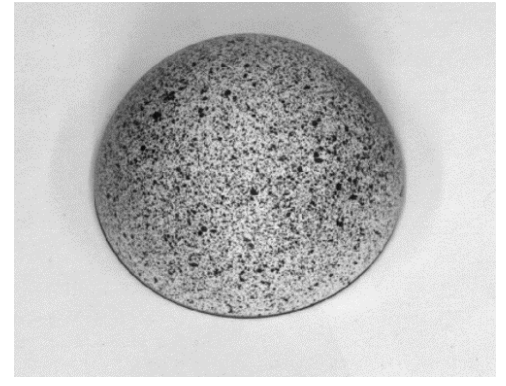

(b) Image taken from above.

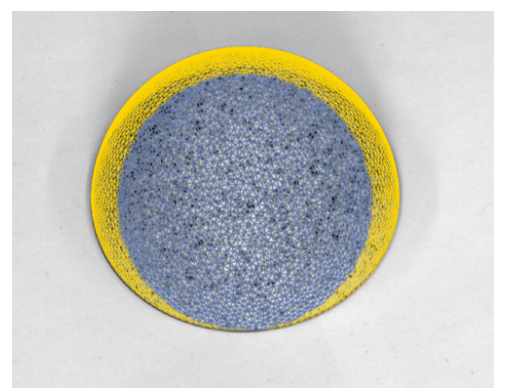

(d) In blue, visible mesh for the camera that took the above picture.

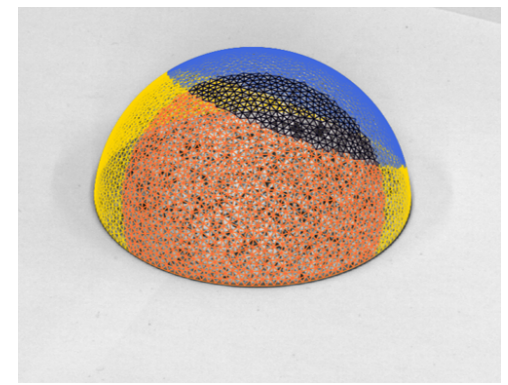

(e) In black, elements that are considered

for the fonctional term associated to the pair of cameras presented above.

Figure 9: Spherical cap, chosen FE mesh (in yellow), and visible parts of the mesh for 2 of the 14 cameras (in red, blue and black). 
value of 0.6. Examples of the elements that are correctly seen by a given camera are shown Figs. $9 \mathrm{c}$ and $9 \mathrm{~d}$, and an example of the elements that are correctly seen by a pair of cameras is given Fig. 9e.

We used a total of three scales, with three image resolutions from $640 \times 512$ to $2560 \times 2048$ pixels using coarse graining and three refinements for the morphing box from $1 \times 1 \times 1$ element to $4 \times 4 \times 4$ elements. $2^{n d}$-degree B-splines were chosen. As explained previously, the FEmesh number of elements and connectivity remain unchanged. A 6571-element triangular FE mesh with first-degree FE basis is used, shown in Fig. 9. For numerical integration, we use Gauss points that are uniformly distributed on the sphere, so that there is at least one Gauss point per pixel.

As explained in section 4.3, the correction is sought along a normal direction. In this example, the surface normal vectors at each FE node are computed as the mean of outwardpointing normal vectors $\underline{n}_{e}$ of the adjacent elements, which is a good approximation of the normal direction at the FE node for regular shapes with fine FE meshes. It is important to note here that unlike in the previous example, $\underline{\tilde{S}_{n}}$ cannot be seen as a displacement of the morphing box control points. $\underline{S}_{n}$ depicts a scalar field inside the morphing box that is the magnitude of the shape correction, and the direction of this correction is only defined at the FE nodes. The same scalar field applied to a different structure would not result in the same shape correction field.

The spherical cap is a surface FE mesh $(d=2)$ embedded in a trivariate patch $(\delta=3)$. As explained in section 2.5, some morphing box control points may not have - or may not have enough - influence on the FE mesh. Therefore, we used the criterion $Q_{i}$ defined in (20) to determine the influence of each control point, and considered the condition number of $\underline{\underline{C}}_{F F D} \stackrel{R_{n}^{T}}{\underline{\underline{H}}} \underline{\underline{R}}_{s} \stackrel{R_{n}}{\underline{\underline{C}}}{ }_{F F D}^{T}$ as described in section 2.5 to spot the less influential control points that make this condition number greater than $10^{8}$. We then removed the corresponding lines of $\underline{\underline{C}}_{F F D}$ as follows:

- 1 removed line over 27 for scale 2 
- 1 removed line over 64 for scale 1

- 37 removed lines over 216 for scale 0 (see Fig. 10)

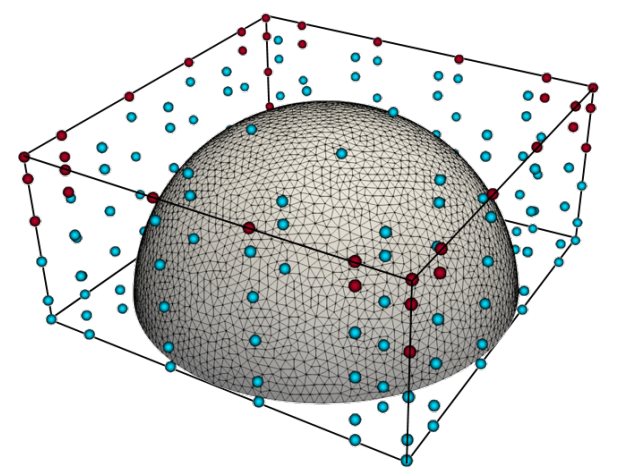

Figure 10: FE mesh embedded in the FFD morphing box at the finest scale, with $4 \times 4 \times 4$ spline elements. The dots are the control points of the morphing box. The red dots are the 37 control points that do not have enough influence, and the blue dots are the remaining dofs.

We obtained the results shown in Fig. 11. More precisely, Fig. 11d shows the results with no regularization at scale 0 . One-element-wavelength noise appears in this case, with unrealistic values at some FE nodes. We observe in Figs. 11a, 11b and 11c that the FFD regularization successfully smooths the shape correction field.

All tested image sets give similar results. The correction on the top of the spherical cap is small, but the size of the spherical cap base seems to be initially underestimated (about $0.27 \mathrm{~mm}$ on the base radius). This result is validated by a shape measurement with the laser scanner Handyscan developed by Creaform3D. The points measured by the laser scan describe a sphere, and the radius of the least-square best fitting sphere on the laser scan data points is slightly greater than the radius used for our initial FE mesh. The correction we measure on Fig. 11 is schematized on Fig. 12.

The laser scan measured points best fitting sphere has a radius of $38.885 \mathrm{~mm}$. We also calculated the least-square best fitting sphere to our measured shape (showed Fig. 11a) by considering the Gauss points, which are the points where graylevel is measured to correct the shape in the modified Gauss-Newton procedure. This sphere has a radius of $38.873 \mathrm{~mm}$, which corresponds to a $0.03 \%$ error on the radius if we take the laser measure as a reference. 


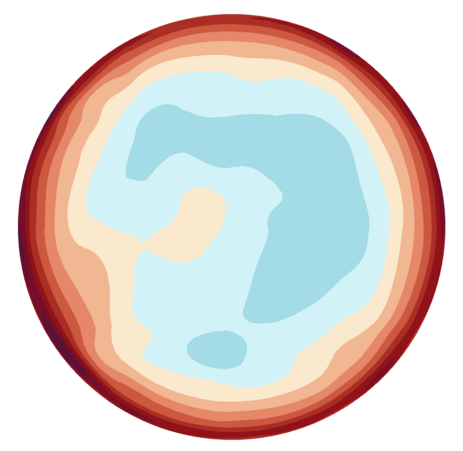

(a) Results with a first set of 14 images.

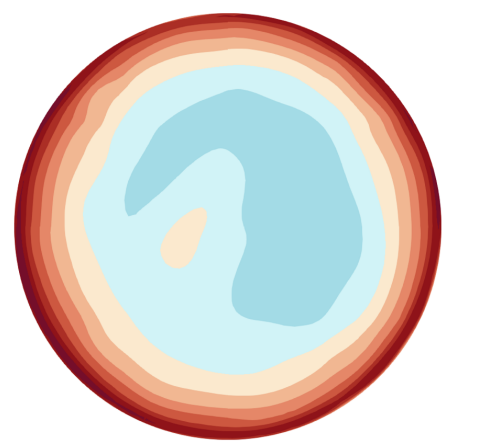

(c) Results with all 50 images.

$$
-2.7 e-04
$$
(e) Color scale for the shape correction along the outer-pointing normal (in $\mathrm{mm}$ ). $2.7 e-04$

0

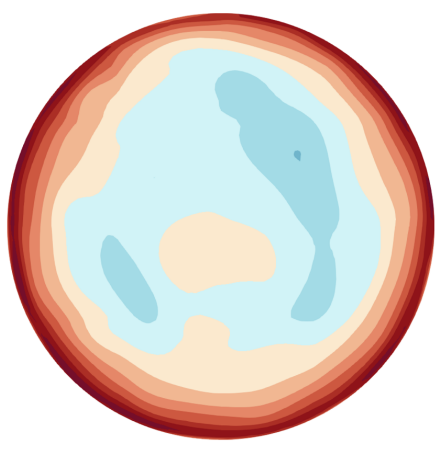

(b) Results with a second similar set of 14 images.

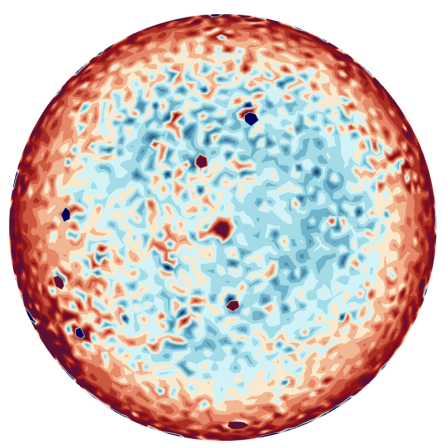

(d) Results with a first set of 14 images, no regularization at scale 0 .

Figure 11: Shape measurement on the spherical cap. The spherical cap is seen from below.

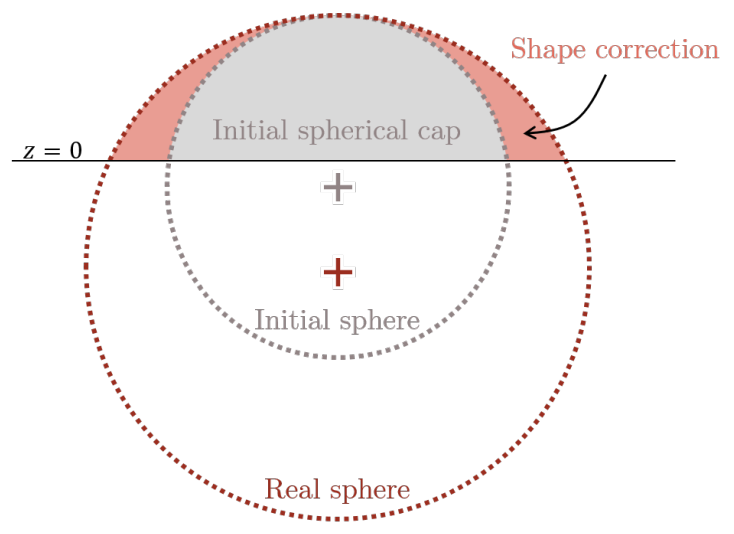

Figure 12: Measured shape correction schematization. 
We also compared the sphericity defects of the FFD-measured shape and the laser-measured shape. For each gaussian point $\underline{M}_{i}$ (resp. measured point for the laser scan), we defined this sphericity defect as $d\left(\underline{M}_{i}, \underline{O}\right)-R$ with $\underline{O}$ the center of the least-square best-fitting sphere and $R$ its radius. We obtained the mean and standard deviation values given table 1 , which shows that our method gives a correct measure of the shape.

\begin{tabular}{|c||c|c|}
\hline & DIC with FFD regularization & laser scan \\
\hline \hline LS radius & $38.87 \mathrm{~mm}$ & $38.89 \mathrm{~mm}$ \\
\hline $\begin{array}{c}\text { Mean of } \\
\text { sphericity defects }\end{array}$ & $2.126 \mathrm{e}-5 \mathrm{~mm}$ & $-1.720 \mathrm{e}-7 \mathrm{~mm}$ \\
\hline $\begin{array}{c}\text { Standard deviation of } \\
\text { sphericity defects }\end{array}$ & $0.02934 \mathrm{~mm}$ & $0.02760 \mathrm{~mm}$ \\
\hline
\end{tabular}

Table 1: Comparison of sphere shape measurement with our method and with the laser scan.

Another shape measurement was carried out with a initial finite element mesh whose geometry is taken a little further from the actual shape.The initial FE mesh has the same center as the previous initial FE mesh, but its radius is decreased by $1 \mathrm{~mm}$, so this spherical cap is a part of a 37.686-mm radius sphere. Figure 13 shows the results after the modified Gauss-Newton minimization. FFD regularization allows finding the right correction, with a 1-mm correction on all the spherical cap and the expected additional $0.27-\mathrm{mm}$ correction near the base of the structure (see Fig. 13b). Without regularization, some FE nodes fail to measure the shape correctly and even take unrealistic correction values.

These results show the capacity of the multiscale FFD regularization to be applied, not only to 2D initial FE meshes, but meshes of any dimension, and with any type of FE elements. Using splines helps obtaining smooth, more realistic shapes, and the proposed regularization method appears robust to initialisation compared to a result without regularization (see Fig. 13). 


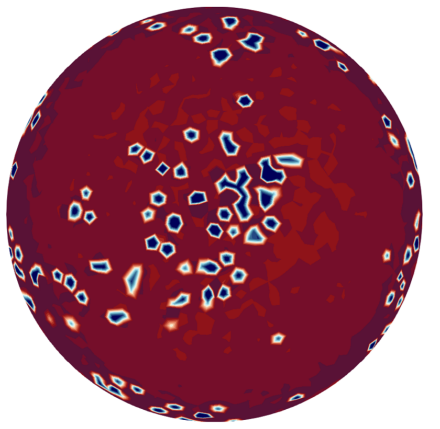

(a) No regularization at scale 0 .

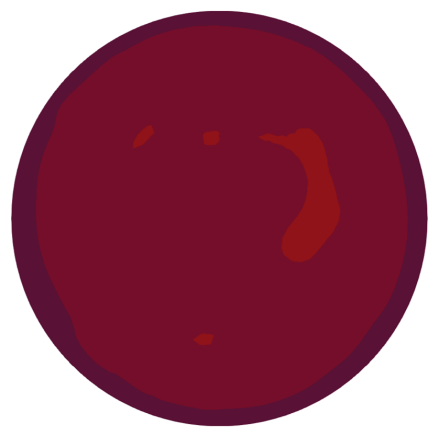

(b) FFD regularization at scale 0 . $1.3 e-03$

(c) Color scale for the shape correction along the outer-pointing normal.

Figure 13: Spherical cap, initialization with a radius that is $1 \mathrm{~mm}$ smaller than in the first experience.

\section{Conclusions}

A non-invasive FFD-based regularization method has been proposed for general full-field measurement in experimental solid mechanics. FE meshes that are used in FE-DIC for easy comparison with simulation results are usually very fine and thus contain too many dof compared to the available amount of data, which makes the corresponding inverse problems highly ill-posed. Embedding the mesh into a cuboid spline morphing box, similarly as in shape optimization for instance [34, 45, 8], offered a way of decoupling the design space from that of the input FE mesh. Indeed, each nodal FE dof of the measured field is controlled by a spline field that is defined over much less control points. In other words, the deformation of the FE mesh follows the smooth spline deformation of the morphing box. This regularization method showed competitive advantages in the experimental mechanics community compared to other published methods. First, the regularization length directly derives from the morphing box refinement, which can easily be set to a desired value thanks to the multilevel properties of B-splines. Then, it can be adapted to any kind of measured field, without any a priori physical knowledge. In this paper, applications to a displacement field measurement and to shape measurements have been shown. Moreover, the FFD regularization method released the constraints imposed by a CAD-based method, which are the need of 
an input boundary-fitted spline mesh and, in case it is made non-invasive from standard FE following [13], of an input FE mesh that has to encompass a spline parametrization. Indeed, the proposed FFD regularization allows to deal with any structure topology and any FE elements type or size. The choice of the FE mesh is independent of the FFD regularization method. The method can also be applied to structures and fields of any dimension, as has been shown in the three given examples with 2D and 3D fields.

Formally, the FFD regularization can be seen as a discretization of a fictitious domain approach $[65,74,60]$. Consequently, it has similar problems to address, as the handling of conditioning problems caused by non-influential control points over the region of interest, which led us to the definition of a criterion to quantify the influence of a control variable. For this purpose, we extended the criteria emanating from the current practice in fictitious domain methods to be adapted to the discretized aspect of our method and to the possible dimension differences between the studied FE-meshed structure and the morphing box. To the best of our knowledge, this is the first time that such a treatment is applied in the general field of FFD.

From a practical point of view, the proposed FFD regularization method can be seen as the projection of the DIC problem, which is originally solved in a FE space, onto a reduced, smoother space. We gave the matrix form of the projection from the FE space to the spline-controlled space. Its computation is easy and it has the advantage of being sparse. This explicit projection matrix allows the use of a standard FE-DIC software in a non-invasive way, which makes this method easily applicable. For shape measurement, we proposed a novel double projection approach to avoid undesired tangential corrections and help the algorithm convergence. In this case, the morphing box controls a scalar field, and the correction direction is defined at the FE nodes and freely chosen, thus widening the range of possible corrections.

We illustrated our method with the analysis of real images coming from three different experiments. The first one consisted in measuring a 2D displacement field on a planar bending beam. A comparison with the standard Tikhonov regularization showed the high potential of the FFD method to strongly regularize without adding artefacts on the resulting 
measured field. The second example validated the hybridation of a $3 \mathrm{D}$ shape measurement problem with a bivariate FFD morphing box. The superiority of the proposed method over standard CAD-based method was highlighted. The last example showed the capacity of the FFD method to be applied for the shape measurement of a more complex 3D geometry. Results of a quality comparable to that of a laser scanner were presented. The robustness to initialization was also demonstrated.

The developed method proved its efficiency for the measurement of relatively regular fields and shapes. It may be easily applied to less regular shapes and extended to less regular fields by defining $C^{0}$ lines in the FFD patch, or by creating a multi-patch FFD morphing box with potentially $C^{-1}$ lines between them, which could be adapted to measuring the propagation of cracks [58]. Hierarchical splines [38, 74] could also be used to allow local refinement, which would be particularly adapted to multiscale DIC experiment [51].

\section{Acknowledgements}

Thanks to Raphaël Fouque for his precious advice and ideas throughout this work and for his help with the calibration of the setup.

\section{References}

[1] Michele Andreoli, Janka Ales, and Jean-Antoine Désidéri. Free-form-deformation parameterization for multilevel 3D shape optimization in aerodynamics. 2003.

[2] Ruben Balcaen, PL Reu, Pascal Lava, and Dimitri Debruyne. Stereo-DIC uncertainty quantification based on simulated images. Experimental Mechanics, 57(6):939-951, 2017.

[3] Eric Bardinet, Laurent D Cohen, and Nicholas Ayache. A parametric deformable model to fit unstructured 3D data. Computer vision and image understanding, 71(1):39-54, 1998.

[4] Brian K Bay, Tait S Smith, David P Fyhrie, and Malik Saad. Digital volume correlation: threedimensional strain mapping using X-ray tomography. Experimental mechanics, 39(3):217-226, 1999.

[5] Benoît Beaubier, J-E Dufour, François Hild, Stéphane Roux, Sylvain Lavernhe, and Karine LavernheTaillard. CAD-based calibration and shape measurement with stereoDIC. Experimental Mechanics, 54 (3):329-341, 2014. 
[6] Gilles Besnard, François Hild, and Stéphane Roux. "Finite-element" displacement fields analysis from digital images: application to Portevin-Le Châtelier bands. Experimental Mechanics, 46(6):789-803, 2006.

[7] Kai-Uwe Bletzinger. A consistent frame for sensitivity filtering and the vertex assigned morphing of optimal shape. Structural and Multidisciplinary Optimization, 49(6):873-895, 2014.

[8] Kai-Uwe Bletzinger. Shape optimization. Encyclopedia of Computational Mechanics Second Edition, pages 1-42, 2017.

[9] Michael J Borden, Michael A Scott, John A Evans, and Thomas JR Hughes. Isogeometric finite element data structures based on Bézier extraction of NURBS. International Journal for Numerical Methods in Engineering, 87(1-5):15-47, 2011.

[10] Vincent Braibant and Claude Fleury. Shape optimal design using B-splines. Computer Methods in Applied Mechanics and Engineering, 44(3):247-267, 1984.

[11] Peng Cheng, Michael A Sutton, Hubert W Schreier, and Stephen R McNeill. Full-field speckle pattern image correlation with B-spline deformation function. Experimental mechanics, 42(3):344-352, 2002.

[12] Elaine Cohen, Tom Lyche, and Richard Riesenfeld. Discrete B-splines and subdivision techniques in computer-aided geometric design and computer graphics. Computer graphics and image processing, 14 (2):87-111, 1980 .

[13] Guillaume Colantonio, Morgane Chapelier, Robin Bouclier, J-C Passieux, and Eduard Marenić. Noninvasive multilevel geometric regularization of mesh-based three-dimensional shape measurement. International Journal for Numerical Methods in Engineering, 121(9):1877-1897, 2020.

[14] J.-A. Cottrell, T.-J.-R Hughes, and A. Reali. Studies of refinement and continuity in isogeometric structural analysis. Computer Methods in Applied Mechanics and Engineering, 196:4160-4183, 09 2007.

[15] John-Eric Dufour, Benoît Beaubier, François Hild, and Stéphane Roux. CAD-based displacement measurements with stereo-DIC. Experimental Mechanics, 55(9):1657-1668, 2015.

[16] John-Eric Dufour, Sylvain Leclercq, Julien Schneider, Stéphane Roux, and François Hild. 3D surface measurements with isogeometric stereocorrelation-application to complex shapes. Optics and Lasers in Engineering, 87:146-155, 2016.

[17] Régis Duvigneau. Adaptive parameterization using free-form deformation for aerodynamic shape optimization. Research Report INRIA, 2006.

[18] Thomas Elguedj, Julien Réthoré, and Aurélien Buteri. Isogeometric analysis for strain field measurements. Computer Methods in Applied Mechanics and Engineering, 200(1-4):40-56, 2011.

[19] David Etievant, Yann Quinsat, François Thiebaut, and François Hild. A modal approach for shape defect measurement based on global stereocorrelation. Optics and Lasers in Engineering, 128:106030, 
2020.

[20] Roberto Fedele, Luca Galantucci, and Antonia Ciani. Global 2D digital image correlation for motion estimation in a finite element framework: a variational formulation and a regularized, pyramidal, multigrid implementation. International Journal for Numerical Methods in Engineering, 96(12):739-762, 2013.

[21] Matthias Firl, Roland Wüchner, and Kai-Uwe Bletzinger. Regularization of shape optimization problems using FE-based parametrization. Structural and Multidisciplinary Optimization, 47(4):507-521, 2013.

[22] Dorian Garcia. Mesure de formes et de champs de déplacements tridimensionnels par stéréo-corrélation d'images. PhD thesis, 2001.

[23] Dorian Garcia and Jean-José Orteu. 3D deformation measurement using stereo-correlation applied to experimental mechanics. In Proceedings of the 10th FIG international symposium deformation measurements, pages 19-22, 2001.

[24] LA Gomes Perini, J-C Passieux, and J-N Périé. A multigrid PGD-based algorithm for volumetric displacement fields measurements. Strain, 50(4):355-367, 2014.

[25] Peter Hansbo, Mats G Larson, and Karl Larsson. Cut finite element methods for linear elasticity problems. In Geometrically unfitted finite element methods and applications, pages 25-63. Springer, 2017.

[26] François Hild and Stéphane Roux. Comparison of local and global approaches to digital image correlation. Experimental Mechanics, 52(9):1503-1519, 2012.

[27] T Hirschler, Robin Bouclier, A Duval, T Elguedj, and Joseph Morlier. The embedded isogeometric Kirchhoff-Love shell: From design to shape optimization of non-conforming stiffened multipatch structures. Computer Methods in Applied Mechanics and Engineering, 349:774-797, 2019.

[28] Thibaut Hirschler, Robin Bouclier, Arnaud Duval, Thomas Elguedj, and Joseph Morlier. Isogeometric sizing and shape optimization of thin structures with a solid-shell approach. Structural and Multidisciplinary Optimization, 59(3):767-785, 2019.

[29] Majid Hojjat, Electra Stavropoulou, and Kai-Uwe Bletzinger. The vertex morphing method for nodebased shape optimization. Computer Methods in Applied Mechanics and Engineering, 268:494-513, 2014.

[30] Berthold KP Horn and Brian G Schunck. Determining optical flow. In Techniques and Applications of Image Understanding, volume 281, pages 319-331. International Society for Optics and Photonics, 1981.

[31] Thomas JR Hughes, John A Cottrell, and Yuri Bazilevs. Isogeometric analysis: CAD, finite elements, NURBS, exact geometry and mesh refinement. Computer Methods in Applied Mechanics and Engi- 
neering, 194(39-41):4135-4195, 2005.

[32] Elizabeth MC Jones, Mark A Iadicola, et al. A good practices guide for digital image correlation. International Digital Image Correlation Society, 10, 2018.

[33] Alvaro Jorge-Peñas, Alicia Izquierdo-Alvarez, Rocio Aguilar-Cuenca, Miguel Vicente-Manzanares, José Manuel Garcia-Aznar, Hans Van Oosterwyck, Elena M de Juan-Pardo, Carlos Ortiz-de Solorzano, and Arrate Muñoz-Barrutia. Free form deformation-based image registration improves accuracy of traction force microscopy. PloS one, 10(12):e0144184, 2015.

[34] Gaetan Kenway, Graeme Kennedy, and Joaquim Martins. A CAD-free approach to high-fidelity aerostructural optimization. In 13th AIAA/ISSMO multidisciplinary analysis optimization conference, page $9231,2010$.

[35] Gaetan KW Kenway and Joaquim RRA Martins. Multipoint high-fidelity aerostructural optimization of a transport aircraft configuration. Journal of Aircraft, 51(1):144-160, 2014.

[36] J Kiendl, R Schmidt, R Wüchner, and K-U Bletzinger. Isogeometric shape optimization of shells using semi-analytical sensitivity analysis and sensitivity weighting. Computer Methods in Applied Mechanics and Engineering, 274:148-167, 2014.

[37] Josef Kiendl. Isogeometric analysis and shape optimal design of shell structures. PhD thesis, Technische Universität München, 2011.

[38] SM Kleinendorst, JPM Hoefnagels, CV Verhoosel, and AP Ruybalid. On the use of adaptive refinement in isogeometric digitalimage correlation. International Journal for Numerical Methods in Engineering, 104(10):944-962, 2015.

[39] Toni Lassila and Gianluigi Rozza. Parametric free-form shape design with PDE models and reduced basis method. Computer Methods in Applied Mechanics and Engineering, 199(23-24):1583-1592, 2010.

[40] Chau Le, Tyler Bruns, and Daniel Tortorelli. A gradient-based, parameter-free approach to shape optimization. Computer Methods in Applied Mechanics and Engineering, 200(9-12):985-996, 2011.

[41] H. Leclerc, J.-N. Périé, F. Hild, and S. Roux. Digital Volume Correlation: What are the limits to the spatial resolution? Mechanics \& Industry, 13(6):361-371, november 2012.

[42] Byung-Gook Lee and Yunbeom Park. Degree elevation of NURBS curves by weighted blossom. Korean Journal of Computational \& Applied Mathematics, 9(1):151-165, 2002.

[43] G. Legrain. A NURBS enhanced extended finite element approach for unfitted CAD analysis. Computational Mechanics, 52:913-929, 2013.

[44] Bruce D Lucas, Takeo Kanade, et al. An iterative image registration technique with an application to stereo vision. IJCAI paper, 1981.

[45] Zhoujie Lyu and Joaquim RRA Martins. Aerodynamic shape optimization of an adaptive morphing trailing-edge wing. Journal of Aircraft, 52(6):1951-1970, 2015. 
[46] Benjamin Marussig and Thomas JR Hughes. A review of trimming in isogeometric analysis: challenges, data exchange and simulation aspects. Archives of computational methods in engineering, 25(4):10591127, 2018.

[47] Florent Mathieu, Hugo Leclerc, François Hild, and Stéphane Roux. Estimation of elastoplastic parameters via weighted FEMU and integrated-DIC. Experimental Mechanics, 55(1):105-119, 2015.

[48] Attila P Nagy, Samuel T IJsselmuiden, and Mostafa M Abdalla. Isogeometric design of anisotropic shells: optimal form and material distribution. Computer Methods in Applied Mechanics and Engineering, 264:145-162, 2013.

[49] Niels Olhoff, Martin P Bendsøe, and John Rasmussen. On CAD-integrated structural topology and design optimization. Computer Methods in Applied Mechanics and Engineering, 89(1-3):259-279, 1991.

[50] J-C Passieux and J-N Périé. High resolution digital image correlation using proper generalized decomposition: PGD-DIC. International Journal for Numerical Methods in Engineering, 92(6):531-550, 2012.

[51] J-C Passieux, Florian Bugarin, Christoph David, J-N Périé, and Laurent Robert. Multiscale displacement field measurement using digital image correlation: Application to the identification of elastic properties. Experimental Mechanics, 55(1):121-137, 2015.

[52] J.-C. Passieux, R. Bouclier, and J.-N. Périé. A space-time PGD-DIC algorithm: Application to 3D mode shapes measurements. Experimental Mechanics, 58(7):1195-1206, 2018.

[53] Jean-Charles Passieux and Robin Bouclier. Classic and inverse compositional Gauss-Newton in global DIC. International Journal for Numerical Methods in Engineering, 119(6):453-468, 2019.

[54] Jean Noël Périé, Hugo Leclerc, Stéphane Roux, and François Hild. Digital image correlation and biaxial test on composite material for anisotropic damage law identification. International journal of solids and structures, 46(11-12):2388-2396, 2009.

[55] Les Piegl and Wayne Tiller. The NURBS book. Springer Science \& Business Media, 2012.

[56] J-E Pierré, J-C Passieux, and J-N Périé. Finite element stereo digital image correlation: framework and mechanical regularization. Experimental Mechanics, 57(3):443-456, 2017.

[57] Ernst Rank, Martin Ruess, Stefan Kollmannsberger, Dominik Schillinger, and Alexander Düster. Geometric modeling, isogeometric analysis and the finite cell method. Computer Methods in Applied Mechanics and Engineering, 249:104-115, 2012.

[58] J Réthoré. Automatic crack tip detection and stress intensity factors estimation of curved cracks from digital images. International Journal for Numerical Methods in Engineering, 103(7):516-534, 2015.

[59] Julien Réthoré. A fully integrated noise robust strategy for the identification of constitutive laws from digital images. International Journal for Numerical Methods in Engineering, 84(6):631-660, 2010.

[60] Ali Rouwane, Robin Bouclier, Jean-Charles Passieux, and Jean-Noël Périé. Adjusting fictitious domain 
parameters for fairly priced image-based modeling: Application to the regularization of Digital Image Correlation. Computer Methods in Applied Mechanics and Engineering, 373:113507, 2021.

[61] Stéphane Roux and François Hild. Stress intensity factor measurements from digital image correlation: post-processing and integrated approaches. International journal of fracture, 140(1-4):141-157, 2006.

[62] Daniel Rueckert, Luke I Sonoda, Carmel Hayes, Derek LG Hill, Martin O Leach, and David J Hawkes. Nonrigid registration using free-form deformations: application to breast MR images. IEEE transactions on medical imaging, 18(8):712-721, 1999.

[63] Alexei Sacharow, Tobias Surmann, and Dirk Biermann. Adaptive free-form deformation for the modification of CAD/CAM data. In ADVCOMP 2011: The Fifth International Conference on Advanced Engineering Computing and Applications in Sciences, 2011.

[64] Alexei Sacharow, Sven Odendahl, A Peuker, Dirk Biermann, Tobias Surmann, and Andreas Zabel. Iterative, simulation-based shape modification by free-form deformation of the NC programs. Advances in Engineering Software, 56:63-71, 2013.

[65] D. Schillinger and M. Ruess. The finite cell method: A review in the context of higher-order structural analysis of CAD and image-based geometric models. Archives of Computational Methods in Engineering, 22(3):391-455, 2015.

[66] Dominik Schillinger, Praneeth K Ruthala, and Lam H Nguyen. Lagrange extraction and projection for NURBS basis functions: A direct link between isogeometric and standard nodal finite element formulations. International Journal for Numerical Methods in Engineering, 108(6):515-534, 2016.

[67] Thomas W Sederberg and Scott R Parry. Free-form deformation of solid geometric models. In Proceedings of the 13th annual conference on Computer graphics and interactive techniques, pages 151-160, 1986.

[68] Aristeidis Sotiras, Christos Davatzikos, and Nikos Paragios. Deformable medical image registration: A survey. IEEE transactions on medical imaging, 32(7):1153-1190, 2013.

[69] Yaofeng Sun, John HL Pang, Chee Khuen Wong, and Fei Su. Finite element formulation for a digital image correlation method. Applied optics, 44(34):7357-7363, 2005.

[70] Michael A Sutton, WJ Wolters, WH Peters, WF Ranson, and SR McNeill. Determination of displacements using an improved digital correlation method. Image and vision computing, 1(3):133-139, 1983.

[71] Michael A Sutton, Stephen R McNeill, Jeffrey D Helm, and Yuh J Chao. Advances in two-dimensional and three-dimensional computer vision. In Photomechanics, pages 323-372. Springer, 2000.

[72] Tobias Teschemacher, AM Bauer, Thomas Oberbichler, Micheal Breitenberger, Riccardo Rossi, Roland Wüchner, and K-U Bletzinger. Realization of CAD-integrated shell simulation based on isogeometric B-Rep analysis. Advanced Modeling and Simulation in Engineering Sciences, 5(1):19, 2018. 
[73] Marie Tirvaudey, Robin Bouclier, Jean-Charles Passieux, and Ludovic Chamoin. Non-invasive implementation of nonlinear isogeometric analysis in an industrial FE software. Engineering Computations, 2019.

[74] C.-V. Verhoosel, G.-J. van Zwieten, B. van Rietbergen, and R. de Borst. Image-based goal-oriented adaptive isogeometric analysis with application to the micro-mechanical modeling of trabecular bone. Computer Methods in Applied Mechanics and Engineering, 284:138-164, 2015.

[75] Wolfgang A Wall, Moritz A Frenzel, and Christian Cyron. Isogeometric structural shape optimization. Computer Methods in Applied Mechanics and Engineering, 197(33-40):2976-2988, 2008.

[76] Jianzhe Wang and Tianzi Jiang. Nonrigid registration of brain MRI using NURBS. Pattern Recognition Letters, 28(2):214-223, 2007.

[77] Lukas Wittevrongel, Pascal Lava, Stepan Vladimirovitch Lomov, and Dimitri Debruyne. A self adaptive global digital image correlation algorithm. Experimental Mechanics, 55(2):361-378, 2015.

[78] Zhiyong Xie and Gerald E Farin. Image registration using hierarchical B-splines. IEEE Transactions on visualization and computer graphics, 10(1):85-94, 2004. 